\title{
NON-COMMUTATIVE AND COMMUTATIVE INTEGRABILITY OF GENERIC TODA FLOWS IN SIMPLE LIE ALGEBRAS
}

\author{
M.I. GeKhTMAn ${ }^{\star \dagger}$ AND M.Z. ShaPIRO ${ }^{\star \star}$ \\ * Department of Mathematics, \\ The University of Michigan, \\ Ann Arbor, USA \\ e-mail: gekhtman@math.lsa.umich.edu \\ ${ }^{\star \star}$ Matematiska Institutionen, KTH \\ Stockholm, Sweden \\ e-mail:mshapiro@math.kth.se
}

\section{INTRODUCTION}

1.1. The Toda lattice equation introduced by Toda as a Hamilton equation describing the motion of the system of particles on the line with an exponential interaction between closest neighbours gave rise to numerous important generalizations and helped to discover many of the exciting phenomena in the theory of integrable equations. In Flaschka's variables $[\mathrm{F}]$ the finite non-periodic Toda lattice describes an isospectral evolution on the set of tri-diagonal matrices in $s l(n)$. It was explicitly solved and shown to be completely integrable by Moser $[\mathrm{Mo}]$.

In his paper [K1], Kostant comprehensively studied the generalization of Toda lattice that evolves on the set of "tri-diagonal" elements of a semisimple Lie algebra $\mathfrak{g}$ which also turned out to be completely integrable with Poisson commuting integrals being provided by the Chevalley invariants of the algebra. Moreover, in this paper, as well as in the works by Ol'shanetsky and Perelomov [OP], Reyman and Semenov-Tian-Shansky [RSTS1], Symes [Sy], the method of the explicit integration of the Toda equations was extended to the case when evolution takes place on the dual space of the Borel subalgebra of $\mathfrak{g}$. This space is foliated into symplectic leaves of different dimensions and the natural question is what can be said about the Liouville complete integrability of the Toda flows on each of these leaves. In the particular case of generic symplectic leaves in $s l(n)$ the complete integrability was proved by Deift, Li, Nanda and Tomei [DLNT].

This paper was motivated by the work [DLNT] and its Lie algebraic interpretation proposed in [S1], [S2], [EFS]. Our main result is the following

\footnotetext{
${ }^{\dagger}$ Research partially supported by AFOSR grant F49620-96-1-0100
} 
Theorem 1.1. The Toda flows on generic coadjoint orbits in simple Lie algebras are completely integrable.

1.2. Recall that there are several versions of the Toda equation in $\operatorname{sl}(n)$ corresponding to different realizations of the dual $\mathfrak{b}_{+}^{*}$ of a subalgebra $\mathfrak{b}_{+}$of upper triangular matrices. In particular, we can use the trace form to identify $\mathfrak{b}^{*}$ with either linear subspace $S$ of symmetric matrices or affine subspace $\epsilon+\mathfrak{b}_{-}$of lower Hessenberg matrices, where $\mathfrak{b}_{-}$is a subalgebra of lower triangular matrices and $\epsilon=\left(\epsilon_{j k}\right)=\left(\delta_{j+1, k}\right)$. The Toda equation associated with the first realization is called symmetric. Following [EFS], we call the Toda equation on lower Hessenberg matrices the Kostant-Toda equation.

Both the symmetric Toda equation and the Kostant-Toda equation can be written in the Lax form

$$
\dot{X}=[X, B(X)]
$$

where $B(X)$ is a projection of $X$ along the subspace of upper triangular matrices onto a skew-symmetric matrix if $X$ is symmetric or lower triangular matrix if $X$ is lower Hessenberg. In both cases, equation (1.1) is Hamilton equation with a Hamiltonian given by $H(X)=\frac{1}{2} \operatorname{Tr}\left(X^{2}\right)$ and a Poisson structure obtained as a pull-back from the standard Lie-Poisson structure on the dual of the algebra of upper triangular matrices. The Toda equation defines a flow on the symplectic leaf that contains initial data $X_{0}$ and coincide with the orbit through $X_{0}$ of the coadjoint action of the upper triangular group.

When restricted to tridiagonal matrices, equation (1.1), called the Toda lattice, is completely integrable. The maximal Poisson commutative family is provided then by eigenvalues or, equivalently, traces of powers of $X$.

The set of tridiagonal matrices in $S$ or $\epsilon+\mathfrak{b}_{-}$can be viewed as the minimal indecomposable symplectic leaf. Deift, Li, Nanda and Tomei [DLNT] addressed the "maximal" case and proved that the symmetric Toda equation is completely integrable on symplectic leaves containing sufficiently generic full matrices $X$ or, in other words, on generic coadjoint orbits. They considered the minors

$$
P_{k}(\lambda)=\operatorname{det}(X-\lambda \mathrm{Id})_{k}, k=0, \ldots,\left[\frac{(n-1)}{2}\right]
$$

where $(M)_{k}$ is obtained from the matrix $M$ obtained by deleting first $k$ rows and last $k$ columns. If $X$ is generic, i.e. if all lower left $k \times k$ minors of $X(k=1, \ldots,[n / 2])$ are nonzero, then

$$
P_{k}(\lambda)=E_{0 k}\left(\lambda^{n-2 k}+I_{1 k} \lambda^{n-2 k} \cdots+I_{n-2 k, k}\right) .
$$

Functions $I_{j k}, j=2, \ldots, n-2 k$ are invariant under the adjoint action of the parabolic subgroup $\mathbf{P}_{k} \subset S L(n)$ of matrices whose strictly lower triangular parts have all zero entries in the first $k$ columns and last $k$ rows . This observation was used in [DLNT] in order to combine the Adler-Kostant-Symes theorem with Thimm's method [T] of construction of involutive families using nested chains of parabolic subalgebras and to show that $I_{j k}, k=$ $0, \ldots,\left[\frac{(n-1)}{2}\right], j=2, \ldots, n-2 k$ form a maximal involutive family of integrals of the Toda flow, while $I_{1 k}, k=0, \ldots,\left[\frac{(n-1)}{2}\right]$ are coadjoint invariants that completely determine the symplectic leaf. 
Definitions and statements of the previous paragraph remain valid without any changes for the case of the Toda flows on generic coadjoint orbits in $\epsilon+\mathfrak{b}_{-}$even though full symmetric and Kostant-Toda flows are not isomorphic and exhibit different dynamical behaviour. However, in what concerns a complete integrability of the Toda equation, it is lower triangular part of $X$ that really matters. Bearing this in mind, we shall restrict ourselves to the Kostant realization of the Toda flows.

It was observed by Singer $[\mathrm{S} 1, \mathrm{~S} 2]$ that if one represents $(X)_{k}$ as a $2 \times 2$ block matrix $\left(\begin{array}{cc}U_{k} & X_{k} \\ Y_{k} & Z_{k}\end{array}\right)$, where $X_{k}$ and $Y_{k}$ are $(n-2 k) \times(n-2 k)$ and $k \times k$ blocks resp., then $I_{j k}, j=2, \ldots, n-2 k$ are coefficients of the characteristic polynomial $\operatorname{det}\left(\lambda-\phi_{k}\right)$ of the Schur complement

$$
\phi_{k}(X)=X_{k}-U_{k} Y_{k}^{-1} Z_{k}
$$

of $X_{k}$ in $(X)_{k}$. Moreover, it was shown in [S1,S2] that the $k$-chop $\phi_{k}$ considered as a map from $\operatorname{sl}(n)$ into $\operatorname{sl}(n-2 k)$ is Poisson w.r.t. both the Lie-Poisson bracket on and the $R$-matrix bracket whose restriction to $\epsilon+\mathfrak{b}_{-}$provides a Poisson structure for the Toda flow (for definitions, see section 2.3 below). In particular, this gives another proof of the involutivity of $I_{j k}$.

In $[\mathrm{A}]$ it was shown that generic matrix $X$ can be conjugated by an element of the upper triangular group to the following form

$$
A d_{b_{X}} X=\left(\begin{array}{cccccc}
\varkappa_{1} & * & * & \ldots & \ldots & * \\
0 & \varkappa_{2} & * & \ldots & \ldots & * \\
\vdots & \vdots & \ddots & \ddots & \ddots & \vdots \\
0 & 0 & & \ddots & \ddots & \vdots \\
0 & 1 & 0 & \ldots & \varkappa_{2} & * \\
1 & 0 & 0 & \ldots & 0 & \varkappa_{1}
\end{array}\right)
$$

Here the only nonzero entries in the lower triangular part of $A d_{b_{X}} X$ are units on the anti-diagonal, while the diagonal part of $A d_{b_{X}} X$ is symmetric w.r.t the anti-diagonal. The element $b_{X}$ is defined uniquely up to a right multiplication by an invertible diagonal matrix symmetric w.r.t the anti-diagonal. This ambiguity does not affect values of $\varkappa_{1}, \varkappa_{2}, \ldots$ and thus, $\varkappa_{1}, \varkappa_{2}, \ldots$ are coadjoint invariants and (1.5) can be considered as a normal form of a generic coadjoint orbit through $X$. Note that the normal form (1.5) can be obtained in several similar steps, the first one being a conjugation with an upper triangular matrix

$$
\Gamma=\left(\begin{array}{cccccc}
\gamma_{11} & \gamma_{12} & \ldots & \ldots & \ldots & \gamma_{1 n} \\
0 & 1 & 0 & \ldots & 0 & \vdots \\
\vdots & \vdots & \ddots & \ddots & \vdots & \vdots \\
0 & 0 & & \ddots & 0 & \vdots \\
0 & 0 & 0 & \ldots & 1 & \gamma_{n-1, n} \\
0 & 0 & 0 & \ldots & 0 & \gamma_{11}^{-1}
\end{array}\right)
$$


where

$$
\begin{aligned}
\gamma_{11} & =x_{n 1}^{\frac{1}{2}} \\
\gamma_{i n} & =-x_{i 1} / x_{n 1}, \gamma_{n i}=\gamma_{11} x_{n i} / x_{n 1}, i=2, \ldots, n-1 \\
\gamma_{1 n} & =-\frac{\gamma_{11}}{x_{n 1}^{2}} \sum_{i=2}^{n-1} x_{n i} x_{i 1}
\end{aligned}
$$

Then a direct computation shows that

$$
A d_{\Gamma} X=\left(\begin{array}{ccc}
\varkappa_{1} & * & * \\
0 & \phi_{1}(X) & * \\
1 & 0 & \varkappa_{1}
\end{array}\right) .
$$

Thus, the 1-chop $\phi_{1}(X)$ can be constructed via the adjoint action of the Borel subgroup. This simple observation is crucial for our generalization of results of [DLNT], [S1], [S2], [EFS] to the general case of the Toda flows on simple Lie algebras. It suggests that in the general situation one can use the adjoint action of the Borel subgroup and projection on the subalgebra to construct an analogue of the 1-chop

$$
\phi_{1}=\pi_{\mathfrak{g}^{\prime}} \circ A d_{\Gamma}
$$

as a map from a simple Lie algebra $\mathfrak{g}$ into its (semi)simple subalgebra $\mathfrak{g}^{\prime}$ in such a way that this map will respect the Lie-Poisson and $R$-matrix brackets. Also, as we can see, a successive application of such maps to a generic element of $\epsilon+\mathfrak{b}_{-}$allows to construct a normal form similar to (1.5) and thus to recover the unpublished result by Kostant on the structure of generic coadjoint orbits.

1.3. Note, that functions $I_{j k}$ defined by (1.2), (1.3) are so-called parabolic Casimirs, i.e. they are $A d_{\mathbf{P}_{k}}$-invariant functions that depend only on the value of the $k$-chop $\phi_{k}(X)$ or, in other words, they can be obtained form the Chevalley invariants of the Levi component of $\mathbf{P}_{k}$. In the $\operatorname{sl}(n)$ case one can oneself to parabolic Casimirs to construct a maximal involutive family due to the rather accidental fact that the number of independent Chevalley invariants in $\operatorname{sl}(n)$ is equal to the half of the difference between dimensions of generic coadjoint orbits in $\operatorname{sl}(n)$ and in the image of the 1-chop, $\operatorname{sl}(n-2)$. Roughly speaking, this allows to disregard "stars' in (1.7) and to continue constriction of Poisson-commuting integrals using only $\phi_{1}(X)$. However, it was pointed out in [EFS] that this approach does not work in $G_{2}$. This prompted us to use in our construction all parabolic invariants rather then only parabolic Casimirs or, in other words, to use all the data contained in $A d_{\Gamma} X$ before applying $\pi_{\mathfrak{g}^{\prime}}$ in (1.8).

Another phenomenon noticed in $[\mathrm{EFS}]$ is the existence of distinct maximal involutive families for the generic Toda flows, which can be seen already in the $\operatorname{sl}(4)$ case. This led the authors of [EFS] to conjecture that the Toda flows are integrable in the noncommutative sense. Using a reduction to of generic elements of $\epsilon+\mathfrak{b}_{-}$to a normal form and an interplay between the adjoint and coadjoint actions on $\epsilon+\mathfrak{b}_{-}$, we shall prove this conjecture (Theorem 5.1).

The paper is organized as follows. In section 2 we explain notations and collect necessary facts on special subalgebras of a simple algebra $\mathfrak{g}$ that will be used in the sequel. We 
also provide a background on the Toda flows and relevant Poisson structures. In section 3 we define the 1-chop map in the form (1.8) and use it to recover Kostant's result on a structure of generic coadjoint orbits. In section 4 we prove that the 1-chop map is Poisson w.r.t. to both Lie-Poisson and $R$-matrix brackets. In section 5 we prove the theorem on non-commutative integrability of the generic Toda flows and also explain that 1-chop can be viewed as a Hamilton reduction w.r.t. the Poisson non-commutative family of parabolic invariants. The latter result make it possible to prove in section 6 a complete integrability of the Toda flows in $G_{2}$. In section 7 we use what may be called a nonlinear nongeneric version of the Manakov-Mischenko-Fomenko shift of argument method to construct a maximal involutive family of parabolic invariants whose independence is proved in section 8. Finally, in section 9 we conclude the proof of our main theorem.

Acknowledgments. Authors benefited a lot from the stimulating discussions with A.Bloch, P. Deift, N.Ercolani, H.Flaschka, T.Ratiu and S.Singer. We are grateful to S. Singer for sharing with us the notes on Kostant's "cascade construction". This work was initiated while the second author was a visitor at the Department of Mathematics, University of Arizona, to which he wishes to express his gratitude for financial support.

\section{Notations And PRELiminaries}

2.1. Let $\mathfrak{g}$ be a simple Lie algebra over $\mathbb{C}$ of rank $r$ different from $\operatorname{sl}(r+1), \mathbf{G}$ is a corresponding Lie group, $\mathfrak{h}$ is a Cartan subalgebra of $\mathfrak{g}$. We denote by $\Phi$ the root system of $\mathfrak{g}$, by $\Phi^{+}$(resp. $\Phi^{-}$) the set of all positive (resp. negative) roots and by $\alpha_{1}, \ldots, \alpha_{r}$ the basis of simple positive roots. We also fix a Chevalley basis $\left\{e_{\alpha}, \alpha \in \Phi ; h_{i}, i=1, \ldots, r\right\}$ in $\mathfrak{g}$. All properties of the root systems and Chevalley bases that we will need can be found in $[\mathrm{H}]$. Recall, in particular, that for $\alpha, \beta \in \Phi$, the $\beta$-string through $\alpha$ is the the maximal set of roots of the form $\alpha+i \beta, i \in \mathbb{Z}$. Then $i$ ranges from $-p$ to $q$ and $p-q=\langle\alpha \mid \beta\rangle=\frac{2(\alpha, \beta)}{(\beta, \beta)}$.

Let $\mathfrak{b}_{+}, \mathfrak{n}_{+}, \mathfrak{b}_{-}, \mathfrak{n}_{-}$be, respectively, the Borel subalgebra for $h$, its nilradical and their opposites. Corresponding groups are denoted by $\mathbf{B}_{ \pm}, \mathbf{N}_{ \pm}$. We use notations $\pi_{+}=$ $\pi_{\mathfrak{b}_{+}}, \pi_{-}=\pi_{\mathfrak{n}_{-}}, \pi_{\mathfrak{b}_{-}}, \pi_{\mathfrak{n}_{+}}$for the natural projections on $\mathfrak{b}_{+}, \mathfrak{n}_{-}, \mathfrak{b}_{-}, \mathfrak{n}_{+}$.

Following [K1], we define an element $\epsilon=\sum_{i=1}^{r} \alpha_{i}$ and realize $\mathfrak{b}_{+}^{*}$ as an affine subspace $\epsilon+\mathfrak{b}_{-}$. Then the coadjoint action of $\mathbf{B}_{+}$on $\mathfrak{b}_{+}^{*}$ takes the form

$$
A d_{b}^{*} \zeta=\epsilon+\pi_{\mathfrak{b}_{-}} A d_{b}^{-1} \zeta
$$

where $b \in \mathbf{B}_{+}, \zeta \in \epsilon+\mathfrak{b}_{-}$. We denote the coadjoint orbit of $\zeta \in \epsilon+\mathfrak{b}_{-}$by $O_{\zeta}$.

2.2. Let $m$ be the maximal positive root and

$$
h_{m}=\left[e_{m}, e_{-m}\right] .
$$

The following notations will be used throughout the paper: 


$$
\begin{aligned}
& \Phi_{\mathcal{F}}^{ \pm}=\left\{\alpha \in \Phi^{ \pm}:(m, \alpha) \neq 0\right\} \\
& \Phi^{\prime}=\{\alpha \in \Phi:(m, \alpha)=0\} \\
& \mathfrak{g}^{\prime}=\operatorname{Span}\left\{e_{\alpha}: \alpha \in \Phi^{\prime}\right\} \\
& \mathcal{F}_{ \pm}=\operatorname{Span}\left\{e_{\alpha}: \alpha \in \Phi_{\mathcal{F}}^{ \pm}\right\} \\
& \mathcal{P}_{ \pm}=\mathfrak{g}^{\prime}+\mathcal{F}_{ \pm} \\
& \mathcal{V}_{ \pm}=\operatorname{Span}\left\{e_{\alpha}: \alpha \in \Phi_{\mathcal{F}}^{ \pm}, \alpha \neq m\right\} \\
& \tilde{\mathcal{F}}_{ \pm}=\operatorname{Span}\left\{\mathcal{F}_{ \pm}, h_{m}\right\}
\end{aligned}
$$

In the rest of the paper every notation followed by ' will have the same meanilng for $\mathfrak{g}^{\prime}$ as the notation without' has for $\mathfrak{g}$.

It follows from definitions above that

$$
\left[e_{ \pm m}, \mathcal{P}_{ \pm}\right]=0,\left[h_{m}, \mathfrak{g}^{\prime}\right]=0
$$

Clearly, $\Phi^{\prime}$ is a reduced root system and $\mathfrak{g}^{\prime}$ is a semisimple subalgebra of $\mathfrak{g}$. The Dynkin diagram of $\Phi^{\prime}$ is obtained by deleting from the Dynkin diagram of $\Phi$ the vertices that are connected with the vertex that corresponds to the minimal root $-m$ in the extended Dynkin diagram of $\Phi$. The rank of $\Phi^{\prime}$ is $r-1$ if $\Phi$ is not of type $A_{r}$ and $r-2$ otherwise (see, e.g. [OV], table 6).

Denote the Killing form on $\mathfrak{g}$ by $\langle$,$\rangle . If \mathfrak{g}=\mathcal{L}_{1}+\mathcal{L}_{2}$ is a decomposition of $\mathfrak{g}$ into the direct sum of linear subspaces, we will identify the dual space $\mathcal{L}_{1}^{*}$ with the subspace $\mathcal{L}_{2}^{\perp}$ orthogonal to $\mathcal{L}_{1}$ w.r.t. the Killing form. In particular,

$$
\mathcal{F}_{ \pm}^{*} \simeq \mathcal{F}_{\mp}, \quad \mathcal{V}_{ \pm}^{*} \simeq \mathcal{V}_{\mp}, \quad \mathcal{P}_{ \pm}^{*} \simeq \mathcal{P}_{\mp}
$$

If $f$ is a smooth function on $\mathcal{L}_{1}$ we define $\nabla f(\xi) \in \mathcal{L}_{2}^{\perp}$ for $\xi \in \mathcal{L}_{1}$ by

$$
\frac{d}{d \varepsilon} f(\xi+\varepsilon \delta \xi) \uparrow_{\varepsilon=0}=\langle\nabla f(\xi), \delta \xi\rangle
$$

The direct sum decomposition

$$
\mathfrak{g}=\mathcal{F}_{-}+\mathcal{F}_{+}+\mathbb{C}\left\{h_{m}\right\}+\mathfrak{g}^{\prime}=\tilde{\mathcal{F}}_{-}+\mathcal{P}_{+}=\mathcal{P}_{-}+\tilde{\mathcal{F}}_{+}
$$

will prove useful in the sequel as will a representation of elements $g \in \mathfrak{g}$ in the form

$$
g=x_{-} e_{-m}+v_{-}+x_{0} h_{m}+v_{+}+x_{+} e_{m}+g^{\prime}
$$

where $v_{ \pm} \in \mathcal{V}_{ \pm}, g^{\prime} \in \mathfrak{g}^{\prime}$. We can define

$$
\pi_{\mathcal{V}_{ \pm}} g=v_{ \pm}, \pi_{\mathfrak{g}^{\prime}} g=g^{\prime}
$$

The properties of $\mathcal{F}_{ \pm}$can be summarized in the following 


\section{Proposition 2.1.}

(1) $\mathcal{F}_{ \pm}$is a nilpotent subalgebra of dimension $2 N+1$.

(2) The following relations hold true

$$
\left[e_{ \pm m}, \mathcal{V}_{\mp}\right]=\mathcal{V}_{ \pm},\left[\mathcal{V}_{ \pm}, \mathcal{V}_{ \pm}\right]=\mathbb{C}\left\{e_{ \pm m}\right\},\left[\mathfrak{g}^{\prime}, \mathcal{V}_{ \pm}\right] \subset \mathcal{V}_{ \pm}
$$

(3)

$$
\left[\mathcal{V}_{+}, \mathcal{V}_{-}\right] \cap\left(\mathcal{V}_{-}+\mathcal{V}_{+}\right)=0
$$

(4) $a d_{\mathfrak{g}^{\prime}}\left\lceil\mathcal{V}_{ \pm}\right.$is an irreducible representation of $\mathfrak{g}^{\prime}$ that preserves a nondegenerate skewsymmetric form $\left\langle a d_{e_{\mp m}}, \cdot\right\rangle$. All weights of this representation are nonzero and all weight spaces are one-dimensional.

Proof. (1) Since $(m, \alpha) \geq 0$ for any $\alpha \in \Phi^{+}$we conclude that $\mathcal{F}_{+}$is a subalgebra. Obviously, it is nilpotent. Moreover, since for $\alpha \in \Phi_{\mathcal{F}}^{+}(m, \alpha)>0$ and $m+\alpha \notin \Phi$, it follows that $m-\alpha$ also belongs to $\Phi_{\mathcal{F}}^{+}$. Roots $\alpha$ and $m-\alpha$ cannot coincide, therefore the dimension of $\mathcal{F}_{+}$is odd.

(2) Let $\alpha \in \Phi_{\mathcal{F}}^{+} \backslash\{m\}$. Then the $m$-string through $\alpha$ contains only $\alpha, \alpha-m$. Clearly, $\quad \alpha-m \in \Phi_{\mathcal{F}}^{-} \backslash\{-m\}$ and the first equality in (2.6) is proved. Moreover, $\langle\alpha \mid m\rangle=\frac{2(\alpha, m)}{(m, m)}=1$. If $\alpha, \beta, \gamma=\alpha+\beta$ all belong to $\Phi_{\mathcal{F}}^{+} \backslash\{m\}$, we have $(m, m)=$ $2(\gamma, m)=2(\alpha+\beta, m)=2(m, m)$ which is absurd. Thus, $\alpha+\beta \in \Phi_{\mathcal{F}}^{+}$if and only if $\alpha+\beta=m$. This proves the second equality in (2.6). The third relation follows from the fact that $(m, \alpha+\beta)=(m, \alpha)>0$ for any $\alpha \in \Phi_{\mathcal{F}}^{+} \backslash\{m\}, \beta \in \Phi^{\prime}$.

(3) follows from $(m, \alpha-\beta)=(m, m)-(m, m)=0$ for any $\alpha, \beta \in \Phi_{\mathcal{F}}^{+} \backslash\{m\}$.

(4) Due to the last relation of $(2.6), \mathfrak{g}^{\prime}$ acts on $\mathcal{V}_{+}$. Since $\mathfrak{g}$ is not of type $A_{n}$, all the roots in $\Phi_{\mathcal{F}}^{+} \backslash\{m\}$ has a form $\alpha=\alpha_{i_{0}}+\sum_{i \neq i_{0}} n_{i} \alpha_{i}$, where $\alpha_{i_{0}}$ is the only simple root connected with $-m$ in the extended Dynkin diagram of $\mathfrak{g}$. It follows that a cyclic subspace of $a d_{\mathfrak{g}^{\prime}}$ generated by $e_{\alpha_{i_{0}}}$ coincides with $\mathcal{V}_{+}$. The weight spaces of $a d_{\mathfrak{g}^{\prime}} \uparrow_{\mathcal{V}_{+}}$are just the root spaces corresponding to different $\alpha \in \Phi_{\mathcal{F}}^{+} \backslash\{m\}$. Finally, the statement about the form $\left\langle a d_{e_{-m}} \cdot, \cdot\right\rangle$ follows from (2.3) and (2.6).

Remark. In the $\operatorname{sl}(n)$ case $\mathcal{F}_{+}$is spanned by matrix elements $e_{1 i}, e_{j n}, i=2, \ldots, n, j=$ $2, \ldots, n-1, \mathfrak{g}^{\prime}$ is $\operatorname{sl}(n-2)$ embedded into $\operatorname{sl}(n)$ as a central $(n-2) \times(n-2)$ block and $a d_{\mathfrak{g}^{\prime}}\left\lceil\mathcal{V}_{+}\right.$has to irreducible components.

Since for any $\alpha \in \Phi_{\mathcal{F}}^{+} \backslash\{m\}$ the $\alpha$-string through $m$ is $m, m-\alpha$, we have (see [H], p. 146) for $v_{ \pm} \in \mathcal{V}_{ \pm}$

$$
\left[e_{m},\left[e_{-m}, v_{+}\right]\right]=v_{+},\left[e_{-m},\left[e_{m}, v_{-}\right]\right]=v_{-} .
$$

2.3. Recall that the Lie-Poisson (Kirillov-Kostant) bracket $\{$,$\} is defined by$

$$
\left\{f_{1}, f_{2}\right\}(g)=\left\langle g,\left[\nabla f_{1}(g), \nabla f_{2}(g)\right]\right\rangle
$$

and $R$-matrix bracket ([RSTS1], [RSTS1], [STS]) $\{,\}_{R}$ is defined by

$$
\begin{aligned}
\left\{f_{1}, f_{2}\right\}_{R}(g) & =\left\langle g,\left[\pi_{+} \nabla f_{1}(g), \pi_{+} \nabla f_{2}(g)\right]-\left[\pi_{-} \nabla f_{1}(g), \pi_{-} \nabla f_{2}(g)\right]\right\rangle \\
= & \left\langle g,\left[\nabla f_{1}(g), \nabla f_{2}(g)\right]-\left[\nabla f_{1}(g), \pi_{-} \nabla f_{2}(g)\right]-\left[\pi_{-} \nabla f_{1}(g), \pi_{-} \nabla f_{2}(g)\right]\right\rangle(2 . \\
= & \left\langle g,-\left[\nabla f_{1}(g), \nabla f_{2}(g)\right]+\left[\nabla f_{1}(g), \pi_{+} \nabla f_{2}(g)\right]+\left[\pi_{+} \nabla f_{1}(g), \pi_{-} \nabla f 2(g)\right]\right\rangle .
\end{aligned}
$$


The Lie-Poisson bracket on $\mathfrak{b}_{+}^{*}$ is defined by

$$
\left\{f_{1}, f_{2}\right\}(\eta)=\eta\left(\left[d f_{1}(g), d f_{2}(g)\right]\right), \eta \in \mathfrak{b}_{+}^{*}
$$

and its pull-back to $\epsilon+\mathfrak{b}_{-}$can be found as

$$
\left\{\tilde{f}_{1}, \tilde{f}_{2}\right\}_{\epsilon+\mathfrak{b}_{-}}(\zeta)=\left\langle\zeta,\left[\pi_{+} \nabla f_{1}(\zeta), \pi_{+} \nabla f_{2}(\zeta)\right]\right\rangle
$$

Note, that $\left(\epsilon+\mathfrak{b}_{-},\{,\}_{\epsilon+\mathfrak{b}_{-}}\right)$is a Poisson submanifold of $\left(\mathfrak{g},\{,\}_{R}\right)$. Symplectic leaves of (2.11) are coadjoint orbits of $\mathbf{B}_{+}$in $\epsilon+\mathfrak{b}_{-}$.

The Hamiltonian $H(\zeta)=\langle\zeta, \zeta\rangle$ generates the Kostant-Toda flow on $\epsilon+\mathfrak{b}_{-}$. The corresponding Hamilton's equation has a Lax form

$$
\dot{\zeta}=\left[\zeta, \pi_{-} \zeta\right]
$$

and can be solved via the factorization method ([K1], [OP], [RSTS1], [Sy]) :

If $\zeta(0)=\zeta_{0}$ is initial condition for $(2.12)$, consider the factorization $\exp \left(t \zeta_{0}\right)=n(t) b(t)$ with $n(t) \in \mathbf{N}_{-}, b(t) \in \mathbf{B}_{+}$. Then

$$
\zeta(t)=b(t) \zeta_{0} b(t)^{-1} .
$$

The important consequence of (2.13) is that a restriction on $\epsilon+\mathfrak{b}_{-}$of any $A d_{\mathbf{B}_{+}}$-invariant function on $\mathfrak{g}$ is an integral of motion of the full Kostant-Toda flow.

In the proposition below we list well-known properties of brackets (2.9)-(2.11) that will be used in the next sections. We suggest [RSTS2] as a reference.

Proposition 2.2. Let $I$ be $A d_{\mathbf{G}}$-invariant and $f_{1}, f_{2}$ be $A d_{\mathbf{B}_{+}}$-invariant functions on $\mathfrak{g}$ and $\tilde{I}, \tilde{f}_{1}, \tilde{f}_{2}$ be their restriction on $\epsilon+\mathfrak{b}_{-}$. Then

$$
\left\{f_{1}, f_{2}\right\}_{R}=\left\{f_{1}, f_{2}\right\}
$$

$$
\left\{\tilde{f}_{1}, \tilde{f}_{2}\right\}_{\epsilon+\mathfrak{b}_{-}}=\left\{f_{1}, f_{2}\right\} \uparrow_{\epsilon+\mathfrak{b}_{-}}
$$

$$
\left\{\tilde{I}, \tilde{f}_{1}\right\}_{\epsilon+\mathfrak{b}_{-}}=0
$$

\section{Generic Coadjoint Orbits}

3.1. Let $g \in \mathfrak{g}$ is such that in the decomposition $(2.5) x_{-} \neq 0$.

Proposition 3.1. For any nonzero $\lambda \in \mathbb{C}$, there exists a unique element $\Gamma \in \exp \left(\tilde{\mathcal{F}}_{+}\right)$ such that

$$
A d_{\Gamma} g=\lambda e_{-m}+\phi_{1}(g)+F_{1}(g):=\lambda e_{-m}+P(g),
$$

where $\phi_{1}(g) \in \mathfrak{g}^{\prime}, F(g) \in \mathcal{F}_{+}$.

Proof. Let $g \in \mathfrak{g}$ be decomposed as in (2.5). According to (2.6), (2.7), for any $w \in \mathcal{V}_{+}$ $a d_{w} g \in \mathfrak{g}-\mathbb{C}\left\{e_{-m}\right\}, a d_{w}^{2} g \in \mathfrak{g}^{\prime}+\tilde{\mathcal{F}}_{+}$. Therefore, a projection of $\exp \left(a d_{w}\right) g$ on $\mathcal{V}_{-}$is equal to $v_{-}+x_{-}\left[w, e_{-m}\right]$. Put $w=x_{-}^{-1}\left[e_{m}, v_{-}\right]$. Then, by $(2.8)$

$$
\tilde{g}=A d_{\exp (w)} g=\exp \left(a d_{w}\right) g=x_{-} e_{-m}+\tilde{x}_{0} h_{m}+\tilde{v}_{+}+\tilde{x}_{+} e_{m}+\tilde{g}^{\prime} .
$$


Now it is not hard to see that for a suitable $\nu \in \mathbb{C}$

$$
\Gamma=\exp \left(\nu h_{m}\right) \exp \left(-\frac{\tilde{x}_{0}}{x_{-}} e_{m}\right) \exp (w)
$$

satisfies (3.1).

The uniqueness of $\Gamma$ follows from the fact that no nonzero element from $\tilde{\mathcal{F}}_{+}$commutes with $e_{-m}$.

Definition 3.1. We call the map $\phi_{1}: \mathfrak{g} \rightarrow \mathfrak{g}^{\prime}$ the 1-chop.

Remarks. 1. In the $\operatorname{sl}(r+1)$ case Definition 3.1 coincides with that of $[\mathrm{S} 1, \mathrm{~S} 2]$. However, (3.1) in this case should read

$$
A d_{\Gamma} g=\lambda e_{-m}+\phi_{1}(g)+F_{1}(g)+\varkappa_{1}(g) h_{0},
$$

where $h_{0}$ is the normalized element $\mathfrak{h}$ which is orthogonal to $h_{m}$ and to the Cartan subalgebra $\mathfrak{h}^{\prime}$ of $\mathfrak{g}^{\prime}$.

2. One can extend the definition of the 1-chop to semisimple algebras by defining $\phi_{1}$ in the way described above on each of the simple components.

3. It follows from a construction of $\Gamma, \phi_{1}, F_{1}$, that "matrix elements" $\left\langle F_{1}(\cdot), e_{-\alpha}\right\rangle, \alpha \in$ $\Phi_{\mathcal{F}}^{+},\left\langle\phi_{1}(\cdot), e_{\gamma}\right\rangle, \gamma \in \Phi^{\prime+}$ considered as functions on $\epsilon+\mathfrak{b}_{-}$are almost everywhere independent.

3.2. The transition from $\Phi$ to $\Phi^{\prime}$ is the first step of Kostant's "cascade construction", which can be found in $[\mathrm{J}],[\mathrm{LW}]$. If one applies this step again to each of the irreducible components $\Phi_{i}^{\prime}$ of $\Phi^{\prime}$, one can define maximal roots $m_{1 i} \in \Phi_{i}^{\prime}$ and root systems $\left(\Phi_{i}^{\prime}\right)^{\prime}$. Continuation of this procedure until it eventually ends enables us to construct a maximal set $\mathfrak{M}=\left\{m, m_{1 i}, \ldots\right\}$ of strongly orthogonal roots in $\Phi^{+}$, i.e. maximal subset of $\Phi^{+}$such that for any two roots in it neither their nor their difference is a root.

Let us enumerate elements of $\mathfrak{M}: \mathfrak{M}=\left\{\beta_{1}=m, \ldots, \beta_{l}\right\}$. In view of the last remarks, we can define 1-chops $\phi_{2}: \mathfrak{g}^{\prime} \rightarrow \mathfrak{g}_{2}=\left(\mathfrak{g}^{\prime}\right)^{\prime}, \phi_{2}: \mathfrak{g}_{2} \rightarrow \mathfrak{g}_{3}=\left(\mathfrak{g}_{2}\right)^{\prime}, \ldots, \phi_{k+1}: \mathfrak{g}_{k} \rightarrow 0$. Then maximal roots of simple components of $\mathfrak{g}, \mathfrak{g}^{\prime}, \ldots, \mathfrak{g}_{k}$ form $\mathfrak{M}$. Note that, by construction, each simple root $\alpha_{i}$ is not orthogonal to exactly one root in $\mathfrak{M}$ and, on the other hand, each $\beta_{j} \in \mathfrak{M}$ is not orthogonal to either one or two simple roots. This observation allows one to describe the number $l=\operatorname{Card}(\mathfrak{M})$ in the following way.

Proposition 3.2. Let $w_{0}$ be the longest element of the Weyl group and s be the number of simple roots which are not invariant under the action of $-w_{0}$. Then

$$
l+\frac{s}{2}=r .
$$

Proof. For every $\beta_{j} \in \mathfrak{M},-w_{0}\left(\beta_{j}\right)=\beta_{j}([\mathrm{LW}]$, Lemma 3.1$)$. Then $\left(\beta_{j},-w_{0}\left(\alpha_{i}\right)\right)=$ $\left(\beta_{j}, \alpha_{i}\right)$, which implies that $-w_{0}$ interchanges $\alpha_{i}, \alpha_{i^{\prime}}$ which are not orthogonal to the same $\beta_{j} \in \mathfrak{M}$ and fixes all other simple roots. The statement follows.

Denote

$$
\begin{aligned}
& \phi^{(j)}=\phi_{j} \circ \phi_{j-1} \circ \cdots \circ \phi_{1}, j=1, \ldots, k \\
& \phi^{(k+1)}=F_{k+1} \circ \phi^{(k)},
\end{aligned}
$$

where $F_{j}, j=1, \ldots, k+1$ can be defined similarly to $(3.1)$. 
Definition 3.2. We call an element $g \in \mathfrak{g}$ generic if maps $\phi^{(j)}, j=1, \ldots, k+1$ are well defined on $g$. A coadjoint orbit $O_{\zeta}$ in $\epsilon+\mathfrak{b}_{-}$is called generic if $\zeta$ is generic.

Let now $\zeta \in \epsilon+\mathfrak{b}_{-}$be generic and let $\epsilon^{\prime}=\sum_{\left(\alpha_{i}, m\right)=0} e_{\alpha_{i}}$. Then $\zeta^{\prime}=\epsilon^{\prime}+\pi_{\mathfrak{b}_{-}} \phi_{1}(\zeta)$ is a generic element in $\mathfrak{g}^{\prime}$.

\section{Proposition 3.3.}

$\operatorname{dim} O_{\zeta}-\operatorname{dim} O_{\zeta^{\prime}}=\operatorname{dim} \tilde{\mathcal{F}}_{+}=2 N+2$

$\operatorname{codim} O_{\zeta}=\operatorname{codim} O_{\zeta^{\prime}}$.

Proof. By Proposition 3.1 there exists unique $\Gamma \in \exp \left(\tilde{\mathcal{F}}_{+}\right)$such that $\zeta_{\lambda}=A d_{\Gamma^{-1}}^{*} \zeta=$ $\lambda e_{-m}+\pi_{\mathfrak{b}_{-}} \phi_{1}(\zeta)+\epsilon$. Then (i) for any $v_{-} \in V_{-} ; x_{-} \neq 0, x_{0} \in \mathbb{C}$ there exists $\xi \in O_{\zeta}$ such that $\pi_{\tilde{\mathcal{F}}_{+}} \xi=x_{-} e_{-m}+v_{-}+x_{0} h_{m} ;$ (ii) $\left(A d_{b}^{*} \zeta_{\lambda}-\zeta_{\lambda}\right) \in \mathfrak{g}^{\prime}$ implies $b \in \mathbf{B}_{+}^{\prime}=\mathbf{B}_{+} \cap \mathbf{G}^{\prime}$.

Therefore, $O_{\zeta^{\prime}} \simeq A d_{\mathbf{B}_{+}^{\prime}}^{*} \zeta_{\lambda}$ and $O_{\zeta}=A d_{\exp \left(\tilde{\mathcal{F}}_{+}\right)}^{*} A d_{\mathbf{B}_{+}^{\prime}}^{*} \zeta_{\lambda} \simeq O_{\zeta^{\prime}} \times \mathbb{C}^{2 N+1} \times \mathbb{C}^{*}$.

Remark. If $\mathfrak{g}$ is $\operatorname{sl}(r+1)$ then $\operatorname{codim} O_{\zeta}-\operatorname{codim} O_{\zeta^{\prime}}=1$, since $\varkappa_{1}$ in $\left(3.1^{\prime}\right)$ is a coadjoint invariant.

In the table below we have listed for all simple algebras the type of $\mathfrak{g}^{\prime}$ and the difference $\Delta$ dim of dimensions of generic coadjoint orbits $O_{\zeta}$ and $O_{\zeta^{\prime}}$.

$\begin{array}{lllllllllc}\mathfrak{g} & A_{n} & B_{n} & C_{n} & D_{n} & E_{6} & E_{7} & E_{8} & F_{4} & G_{2} \\ \mathfrak{g}^{\prime} & A_{n-2} & A_{1}+B_{n-2} & C_{n-1} & A_{1}+D_{n-2} & A_{5} & D_{6} & E_{7} & C_{3} & A_{1} \\ \Delta \operatorname{dim} & 2 n & 4 n-4 & 2 n & 4 n-6 & 22 & 34 & 58 & 16 & 6\end{array}$

Repeatedly applying Propositions $3.1,3.3$ to $\mathfrak{g}^{\prime}, \mathfrak{g}_{2}, \ldots, \mathfrak{g}_{k}$ we recover the following result due to Kostant :

Theorem 3.1. Let $\mathfrak{h}_{0}$ be an orthogonal complement in $\mathfrak{h}$ to $\operatorname{Span}\left\{\left[e_{-\beta}, e_{\beta}\right], \beta \in \mathfrak{M}\right\}$. If $\zeta$ is generic then there exist the unique element $h_{0} \in \mathfrak{h}_{0}$ such that

$$
\zeta_{0}=\sum_{i=1}^{l} e_{-\beta_{i}}+h_{0}+\epsilon \in O_{\zeta}
$$

The codimension of $O_{\zeta}$ in $\epsilon+\mathfrak{b}_{-}$is equal to the dimension of the stabilizer of $\zeta_{0}$ and is $r-l$.

Remark. Invoking Proposition 3.2 we obtain that the codimension of the generic orbit is $s / 2$. This result was obtained by Trofimov in $[\operatorname{Tr} 2]$.

Due to Theorem 3.1 for any generic $\zeta \in \epsilon+\mathfrak{b}_{-}$there exist $b_{\zeta} \in \mathbf{B}_{+}$such that

$$
A d_{b_{\zeta}}^{*} \xi=\zeta_{0}=\sum_{i=1}^{l} e_{-\beta_{i}}+h_{0}+\epsilon
$$

where $h_{0} \in \mathfrak{h}_{0}$. Denote $\mathfrak{h}_{1}=\mathfrak{h}_{0}^{\perp}=\operatorname{Span}\left\{\left[e_{-\beta}, e_{\beta}\right], \beta \in \mathfrak{M}\right\}$ and consider the factorization $\mathbf{T}=\mathbf{T}_{0} \mathbf{T}_{1}$ of the maximal torus $\mathbf{T}$ corresponding to the linear space decomposition $\mathfrak{h}=\mathfrak{h}_{0}+\mathfrak{h}_{1}$. Then a stabilizer of $\zeta_{0}$ under the coadjoint action is $\mathbf{T}_{0}$ and $b_{\zeta}$ is defined uniquely up to a right multiplication by elements of $\mathbf{T}_{0}$. Let $b_{\zeta}=\tilde{b}_{\zeta} t_{\zeta}$ be a factorization 
of $b_{\zeta}$ into the product of unipotent $\tilde{b}_{\zeta}$ and $t_{\zeta} \in \mathbf{T}$. We can make the choice of $b_{\zeta}$ unique by demanding that $t_{\zeta}$ belongs to $\mathbf{T}_{1}$ and thus establish a one-to-one correspondence between $\mathbf{N}_{+} \mathbf{T}_{1}$ and $O_{\zeta}$ :

$$
\mathbf{N}_{+} \mathbf{T}_{1} \ni \tilde{b} t_{1} \leftrightarrow A d_{\left(\tilde{b} t_{1}\right)^{-1}}^{*} \zeta_{0} \in O_{\zeta} .
$$

We finish this section with the following technical lemma.

Lemma 3.1. For $\nu \in \mathfrak{n}_{-},\left[\nu, \zeta_{0}\right] \in \mathfrak{h}_{0}$ implies $\nu=0$.

Proof. Let us represent $\nu$ as a sum $\nu=\nu_{-1}+\nu_{-2}+\cdots$, where $\nu_{-i}$ belongs to the subspace $V_{-i}$ of $\mathfrak{g}$ generated by roots of height $-i$. Then

$$
\begin{aligned}
& {\left[\nu_{-1}, \epsilon\right] \in \mathfrak{h}_{0}} \\
& {\left[\nu_{-2}, \epsilon\right]=-\left[\nu_{-1}, h_{0}\right]} \\
& \ldots \\
& {\left[\nu_{-i-1}, \epsilon\right]=l_{i}\left(\nu_{-1}, \ldots, \nu_{-i}\right)} \\
& \ldots
\end{aligned}
$$

Here

$$
l_{i}\left(\nu_{-1}, \ldots, \nu_{-i}\right)=-\left[\nu_{-i}, h_{0}\right]-\sum_{\operatorname{height}\left(\beta_{j}\right)+k=i}\left[\nu_{-k}, e_{-\beta_{j}}\right] .
$$

It is shown in $[\mathrm{K} 2, \mathrm{~K} 3]$ that $a d_{\epsilon}: V_{-i-1} \rightarrow V_{-i}$ is injective. Therefore, (3.5) implies that $\nu_{-2}, \nu_{-3}, \ldots$ are uniquely determined by $\nu_{-1}$ and, therefore, the number of linearly independent solutions to (3.5) cannot exceed the number of linearly independent $\nu_{-1}$ satisfying the first relation of (3.5), the latter being equal to $\operatorname{dim} \mathfrak{h}_{0}$. Note that for all root systems but $A_{n}, D_{2 n+1}$ and $E_{6}, \operatorname{dim} \mathfrak{h}_{0}=0$. The remaining three cases can be checked by a direct computation.

\section{1-ChOp Is PoISSON}

4.1. Our next objective is to study a behaviour of the 1-chop map $\phi_{1}(g)$ and, more generally, $P(g)$ with respect to Poisson brackets (2.9), (2.10).

First, we compute a variation $\delta P$ :

$$
\delta P=A d_{\Gamma} \delta g-\left[\lambda e_{-m}+P, \delta \gamma\right],
$$

where $\delta \gamma=R_{\Gamma^{-1} *} \delta \Gamma$. Since

$$
\delta P \perp \tilde{\mathcal{F}}_{+},
$$

we have for any $\psi \in \tilde{\mathcal{F}}_{+}$

$$
\left\langle A d_{\Gamma} \delta g, \psi\right\rangle=\left\langle\left[\lambda e_{-m}+P, \delta \gamma\right], \psi\right\rangle=\lambda\left\langle\left[e_{-m}, \delta \gamma\right], \psi\right\rangle .
$$

Let $\delta \gamma=v_{\gamma}+\gamma_{+} e_{m}+\gamma_{0} h_{m}$. Then, by (2.8), for any $v \in \mathcal{V}_{+}$,

$$
\left\langle A d_{\Gamma} \delta g, v\right\rangle=\left\langle\left[A d_{\Gamma} \delta g, e_{m}\right],\left[e_{-m}, v\right]\right\rangle=-\lambda\left\langle v_{\gamma},\left[e_{-m}, v\right]\right\rangle .
$$


Therefore,

$$
v_{\gamma}=\lambda^{-1} \pi_{\mathcal{V}_{+}}\left[e_{m}, A d_{\Gamma} \delta g\right]
$$

To find $\gamma_{0}$, substitute $\psi=e_{m}$ into (4.2) :

$$
\left\langle A d_{\Gamma} \delta g, e_{m}\right\rangle=\lambda \gamma_{0}\left\langle\left[e_{-m}, h_{m}\right], e_{m}\right\rangle=\lambda \gamma_{0}\left\langle h_{m}, h_{m}\right\rangle
$$

Thus,

$$
\gamma_{0}=\lambda^{-1} \frac{\left\langle A d_{\Gamma} \delta g, e_{m}\right\rangle}{\left\langle h_{m}, h_{m}\right\rangle} .
$$

Using (2.6), (4.1), we obtain

$$
\delta P=\pi_{\mathcal{P}_{+}} A d_{\Gamma} \delta g-\left[P, v_{\gamma}+\gamma_{0} h_{m}\right],
$$

where $v_{\gamma}$ and $\gamma_{0}$ are given by (4.3), (4.4).

Now we can compute $\nabla(f \circ P)(g)$ for a function $f \in C^{\infty}\left(\mathcal{P}_{+}\right)$. Indeed,

$$
\begin{aligned}
& \delta(f \circ P)=\langle\nabla f(P), \delta P\rangle \\
& =\left\langle\delta f(P), \pi_{\mathcal{P}_{+}} A d_{\Gamma} \delta g-\lambda^{-1}\left[P, \pi_{\mathcal{V}_{+}}\left[e_{m}, A d_{\Gamma} \delta g\right]-\gamma_{0}\left[P, h_{m}\right]\right\rangle\right. \\
& =\left\langle\nabla f(P), A d_{\Gamma} \delta g\right\rangle-\lambda^{-1}\left\langle\pi_{\mathcal{V}_{-}}[\nabla f(P), P], A d_{\Gamma} \delta g\right\rangle-\lambda^{-1} \frac{\left\langle A d_{\Gamma} \delta g, e_{m}\right\rangle}{\left\langle h_{m}, h_{m}\right\rangle}\left\langle[\nabla f(P), P], h_{m}\right\rangle \\
& =\left\langle A d_{\Gamma}^{-1}\left(\nabla f(P)+\lambda^{-1}\left[e_{m}, \pi_{\mathcal{V}_{-}}[\nabla f(P), P]\right]-\lambda^{-1} \frac{\left\langle[\nabla f(P), P], h_{m}\right\rangle}{\left\langle h_{m}, h_{m}\right\rangle} e_{m}\right), \delta g\right\rangle .
\end{aligned}
$$

Therefore,

$$
\nabla(f \circ P)(g)=A d_{\Gamma}^{-1}\left(\nabla f(P)+\lambda^{-1}\left(v_{f}+x_{f} e_{m}\right)\right)
$$

where

$$
v_{f}=\left[e_{m}, \pi_{\mathcal{V}_{-}}[\nabla f(P), P]\right] \in \mathcal{V}_{+} \text {and } x_{f}=\frac{\left\langle[\nabla f(P), P], h_{m}\right\rangle}{\left\langle h_{m}, h_{m}\right\rangle}
$$

Let us consider a particular case when a function $f$ belongs to $C^{\infty}\left(\mathfrak{g}^{\prime}\right) \subset C^{\infty}\left(\mathcal{P}_{+}\right)$. Then $\nabla f \in \mathfrak{g}^{\prime}$ and $(f \circ P)(g)=\left(f \circ \phi_{1}\right)(g)$. Therefore, by (2.6), (3.6), $v_{f}=0, x_{f}=0, \nabla\left(f \circ \phi_{1}\right)(g) \in \mathcal{P}_{+}, \pi_{\mathfrak{g}^{\prime}} \nabla\left(f \circ \phi_{1}\right)(g)=\nabla f\left(\phi_{1}\right)$ and

$$
\pi_{-} \nabla\left(f \circ \phi_{1}\right)(g)=\pi_{-} \nabla f\left(\phi_{1}\right)=A d_{\Gamma}^{-1} \pi_{-} \nabla f\left(\phi_{1}\right)
$$

These observations enable us to prove the following

Theorem 4.1. The map $\phi_{1}: \mathfrak{g} \rightarrow \mathfrak{g}^{\prime}$, defined by (3.1) is Poisson w.r.t. both Lie-Poisson and $R$-matrix brackets.

Proof. Let $f_{1}, f_{2} \in C^{\infty}\left(\mathfrak{g}^{\prime}\right)$. Then

$$
\begin{aligned}
\left\{f_{1} \circ \phi_{1}, f_{2} \circ \phi_{1}\right\}(g) & =\left\langle A d_{\Gamma} g,\left[\nabla f_{1}\left(\phi_{1}\right)+\lambda^{-1} x_{f_{1}} e_{m}, \nabla f_{2}\left(\phi_{1}\right)+\lambda^{-1} x_{f_{2}} e_{m}\right]\right\rangle \\
& =\left\langle\phi_{1},\left[\nabla f_{1}\left(\phi_{1}\right), \nabla f_{2}\left(\phi_{1}\right)\right]\right\rangle .
\end{aligned}
$$


The second part of the statement can be proved similarly, if one uses (4.6) and the second line of (2.10).

4.2. Next, we compute a push-forward of the Lie-Poisson bracket (2.9) on $\mathfrak{g}$ under the map $P$. For any $f_{1}, f_{2} \in C^{\infty}\left(\mathcal{P}_{+}\right),(3.6)$ implies

$$
\begin{aligned}
\left\{f_{1} \circ P, f_{2} \circ P\right\}(g)= & \left\langle A d_{\Gamma} g,\left[\nabla f_{1}(P)+\lambda^{-1}\left(v_{f_{1}}+x_{f_{2}} e_{m}\right), \nabla f_{2}(p)+\lambda^{-1}\left(v_{f_{2}}+x_{f_{2}} e_{m}\right)\right]\right\rangle \\
= & \lambda^{-1}\left\langle e_{-m},\left[v_{f_{1}}+x_{f_{1}} e_{m}, v_{f_{2}}+x_{f_{2}} e_{m}\right]\right\rangle+\left\langle P,\left[\nabla f_{1}(P), \nabla f_{2}(P)\right]\right\rangle \\
& +\lambda^{-1}\left\langle p,\left[\nabla f_{1}(p), v_{f_{2}}+x_{f_{2}} e_{m}\right]+\left[v_{f_{1}}+x_{f_{1}} e_{m}, \nabla f_{2}(P)\right]\right\rangle \\
= & I+I I+I I I .
\end{aligned}
$$

Here we used the fact that $e_{-m} \perp \mathcal{P}_{-}$. Due to (2.6), (2.8)

$$
\lambda I=\left\langle e_{-m},\left[v_{f_{1}}, v_{f_{2}}\right]\right\rangle
$$

and

$$
\lambda I I I=\left\langle P,\left[\nabla f_{1}(P), v_{f_{2}}\right]+\left[v_{f_{1}}, \nabla f_{2}(P)\right]\right\rangle .
$$

Moreover,

$$
\begin{aligned}
\left\langle e_{-m},\left[v_{f_{1}}, v_{f_{2}}\right]\right\rangle & =\left\langle\left[e_{-m},\left[e_{m}, \pi_{\mathcal{V}_{-}}\left[\nabla f_{1}(P), P\right]\right]\right], v_{f_{2}}\right\rangle \\
& \left.=\left\langle\pi_{\mathcal{V}_{-}}\left[\nabla f_{1}(P), P\right]\right], v_{f_{2}}\right\rangle \\
& =-\left\langle\left[P, \nabla f_{1}(P)\right], v_{f_{2}}\right\rangle .
\end{aligned}
$$

Thus,

$$
\begin{aligned}
\lambda(I+I I I) & =\left\langle P,\left[v_{f_{1}}, \nabla f_{2}(P)\right]\right\rangle \\
& =\left\langle\left[e_{m}, \pi_{\mathcal{V}_{-}}\left[\nabla f_{1}(P), P\right]\right],\left[\nabla f_{2}(P), P\right]\right\rangle \\
& =\left\langle\left[e_{m}, \pi_{\mathcal{V}_{-}}\left[\nabla f_{1}(P), P\right]\right], \pi_{\mathcal{V}_{-}}\left[\nabla f_{2}(P), P\right]\right\rangle .
\end{aligned}
$$

Denote

$$
\begin{aligned}
& \left\{f_{1}, f_{2}\right\}_{0}(p)=\left\langle p,\left[\nabla f_{1}(p), \nabla f_{2}(p)\right]\right\rangle \\
& \left\{f_{1}, f_{2}\right\}_{1}(p)=\left\langle a d_{e_{m}} \pi_{\mathcal{V}_{-}}\left[\nabla f_{1}(p), p\right], \pi_{\mathcal{V}_{-}}\left[\nabla f_{2}(p), p\right]\right\rangle
\end{aligned}
$$

We proved

Proposition 4.1. For any $f_{1}, f_{2} \in C^{\infty}\left(\mathcal{P}_{+}\right)$

$$
\left\{f_{1} \circ P, f_{2} \circ P\right\}=\left\{f_{1}, f_{2}\right\}_{0} \circ P+\lambda^{-1}\left\{f_{1}, f_{2}\right\}_{1} \circ P:=\left\{f_{1}, f_{2}\right\}^{\lambda} \circ P \text {. }
$$

Since $\lambda$ is arbitrary and $P: \mathfrak{g} \rightarrow \mathcal{P}_{+}$is clearly surjective, we obtain as an immediate corollary

Proposition 4.2. $\{,\}_{0}$ and $\{,\}_{1}$ are compatible Poisson brackets. 


\section{Non-Commutative Integrability of Toda Flows}

5.1. The notion of non-commutative integrability we are referring to in this section goes back to results of Nehoroshev $[\mathrm{N}]$. It is more general then linear non-commutative integrability introduced by Mischenko and Fomenko [MiFo1]. For examples and bibliography, we refer to chap. 5 of the book [Fo].

Let $\mathcal{A}$ be a Poisson subalgebra of first integrals of a Hamilton flow on $2 n$-dimensional symplectic manifold.

Definition 5.1. A flow is called integrable in the non-commutative sense if $\mathcal{A}$ is generated by functionally independent functions $f_{1}, \ldots, f_{2 n-m}$ such that $\left\{f_{i}, f_{j}\right\}=0$ for any $i=$ $1, \ldots, m ; j=1, \ldots, 2 n-m$.

Consider a generic coadjoint orbit $O_{\zeta}$ through $\zeta \in \epsilon+\mathfrak{b}_{-}$.

Theorem 5.1. The Poisson subalgebra $\mathcal{A}$ of first integrals of the Toda flow on $O_{\zeta}$ is generated by restrictions to $O_{\zeta}$ of $A d_{\mathbf{B}_{+}}$-invariant functions on $\mathfrak{g}$ and has a functional dimension $\left(\operatorname{dim} O_{\zeta}-r\right)$. Its center is generated by restrictions of the Chevalley invariants of $\mathfrak{g}$. The Toda flow is, therefore, integrable in the non-commutative sense.

Proof. For a generic element $g \in \mathfrak{g}$, we define $b_{g}$ to be equal to $b_{\zeta}$, where $\zeta=\pi_{b_{-}}(g)+\epsilon \in$ $\epsilon+\mathfrak{b}_{-}$and $b_{\zeta}$ is the unique element of $\mathbf{N}_{+} \mathbf{T}_{1}$ satisfying (3.3) .

Note, that if $b_{1}$ is an element from $\mathbf{B}_{+}$and $b_{1}=\tilde{b}_{1} t_{0} t_{1}$ is its factorization into the product of unipotent $\tilde{b}_{1}, t_{0} \in \mathbf{T}_{0}$ and $t_{1} \in \mathbf{T}_{1}$, then

$$
b_{\left(A d_{b} g\right)}=\left(\tilde{b} t_{0} t_{1} \tilde{b}_{\zeta} t_{0}^{-1} t_{1}^{-1}\right) t_{1} t_{\zeta}=b b_{g} t_{0}^{-1} .
$$

For any $\alpha \in \Phi^{+}$, define a function

$$
\varphi_{\alpha}(g)=\left\langle g, A d_{b_{g}} e_{-\alpha}\right\rangle
$$

It follows from (5.1) that $\varphi_{\alpha}(g)$ is semiinvariant under the adjoint action of $\mathbf{B}_{+}$:

$$
\varphi_{\alpha}\left(A d_{b} g\right)=\left\langle g, A d_{b_{g}} A d_{t_{0}}^{-1} e_{-\alpha}\right\rangle=\chi_{\alpha}\left(t_{0}\right) \varphi_{\alpha}(g) .
$$

Moreover, if $\mathbf{k}$ is an integral vector such that $\nu=\sum_{\alpha \in \Phi^{+}} k_{\alpha} \alpha, k_{\alpha} \in \mathbb{Z}$ annihilates $\mathfrak{h}_{0}$ then a function

$$
\theta^{\mathbf{k}}(g)=\Pi_{\alpha \in \Phi^{+}}\left(\varphi_{\alpha}(g)\right)^{k_{\alpha}}
$$

is $A d_{\mathbf{B}_{+}}$-invariant on $\mathfrak{g}$.

The number $K$ of linearly independent vectors $\mathbf{k}$ such that $\nu$ annihilates $\mathfrak{h}_{0}$ is equal to the number of positive roots minus the rank of the matrix $\left(\alpha\left(\eta_{i}\right)\right)_{\alpha \in \Phi^{+}, i=1, \ldots, \operatorname{dim} \mathfrak{h}_{0}}$, where $\eta_{i}, i=1, \ldots, \operatorname{dim} \mathfrak{h}_{0}$ is a basis of $\mathfrak{h}_{0}$. Clearly, this rank is equal to $\operatorname{dim} \mathfrak{h}_{0}=r-l$. Thus

$$
K=\operatorname{Card}\left(\Phi^{+}\right)+l-r=\operatorname{dim} O_{\xi}-r
$$

Now we have to prove that functions $\theta^{\mathbf{k}}$ with linearly independent vectors $\mathbf{k}$ are independent on a generic coadjoint orbit $O_{\zeta}$. Since such $\theta^{\mathbf{k}}$ are obviously independent as polynomials in $\varphi_{\alpha}, \alpha \in \Phi^{+}$, it suffices to show that $\varphi_{\alpha}$ are independent on $O_{\zeta}$. To this 
end, we use the identification (3.4) and estimate the dimension of the linear space generated by differentials at identity of $\varphi_{\alpha}$ considered as functions on $\mathbf{N}_{+} \mathbf{T}_{1}$. Differentiating

$$
\varphi_{\alpha}\left(A d_{(\exp (\varepsilon \delta b))^{-1}}^{*} \zeta_{0}\right)=\left\langle\pi_{\mathfrak{b}_{-}}\left(A d_{\exp (\varepsilon \delta b)} \zeta_{0}\right)+\epsilon, A d_{\exp (\varepsilon \delta b)} e_{-\alpha}\right\rangle \quad, \delta b \in \mathfrak{n}_{+}+\mathfrak{h}_{1}
$$

with respect to $\varepsilon$ at $\varepsilon=0$, we obtain

$$
\delta \varphi_{\alpha}=\left\langle\zeta_{0},\left[\delta b, e_{-\alpha}\right]\right\rangle=\left\langle\delta b,\left[e_{-\alpha}, \zeta_{0}\right]\right\rangle
$$

Thus, linear dependency of $d \varphi_{\alpha}$ at identity is equivalent to existence of nonzero $\nu \in \mathfrak{n}_{-}$ such that $\left[\nu, \zeta_{0}\right] \in \mathfrak{h}_{0}$ which would contradict Lemma 3.1. Thus, the functional dimension of $\mathcal{A}$ is greater or equal then $\left(\operatorname{dim} O_{\zeta}-r\right)$.

To complete the proof, one should notice that if $I_{i}, i=1, \ldots, r$ are independent invariant polynomials on $\mathfrak{g}$, then $I_{i}(\zeta)=I_{i}\left(A d_{b_{\zeta}}^{-1} \zeta\right)$ can be expressed via functions $\theta^{\mathbf{k}}$ and are in involution with any of them (cf. Proposition 2.2). Finally, if there exists either one more integral independent of constructed above or one more element of the center of $\mathcal{A}$ independent of $I_{i}, i=1, \ldots, r$, this is in contradiction with existence of $r$ independent Hamiltonian flows generated by $I_{i}, i=1, \ldots, r$.

Remark. A non-commutative integrability makes possible existence of distinct maximal commutative families for the Toda flows (phenomenon observed in [EFS]) . Another important implication is that, due to Nehoroshev's theorem $[\mathrm{N}]$, the Toda flow is degenerate, i.e. its trajectories lie on invariant manifolds of dimension $r<1 / 2 \operatorname{dim} O_{\zeta}$. In particular, this enables one to reconcile Hamiltonian and gradient structures of the full Toda flows. This aspect is explored in the forthcoming paper [BG].

5.2. Theorem 5.1 allows, in principle, to construct a maximal involutive family of smooth almost everywhere independent first integrals of the Toda flow (cf. [N], [Fo], chap. $5)$. However, if we are looking for a maximal family of rational integrals, it may be helpful to restrict ourselves to a subalgebra of $\mathcal{A}$ by imposing additional symmetry conditions.

Let $\mathbf{P}$ be the parabolic subgroup that stabilizes the weight $m$ (the corresponding subalgebra is $\left.\mathcal{P}_{+}+\mathbb{C}\left\{h_{m}\right\}\right)$. It is easy to see that if $f_{1}$ is an $A d_{\mathbf{P}}$-invariant function on $\mathfrak{g}$ and $f_{2}$ is such that $\nabla f_{2}(g) \in \mathcal{P}_{+}+\mathbb{C}\left\{h_{m}\right\}$, then $\left\{f_{1}, f_{2}\right\}=0$. In particular, we have

Lemma 5.1. If $f_{1}$ is an Ad $d_{\mathbf{P}}$-invariant function on $\mathfrak{g}$ and $f_{2} \in C^{\infty}\left(\mathfrak{g}^{\prime}\right)$, then

$$
\left\{f_{1}, f_{2} \circ \phi_{1}\right\}=0
$$

Recall that, due to Proposition 2.1, the adjoint action of $\mathbf{G}^{\prime}$ preserves $\mathcal{P}_{+}$.

Lemma 5.2. If $f$ is an $A d_{\mathbf{G}^{\prime}}$-invariant function on $\mathcal{P}_{+}$, then $f \circ P$ is an $A d_{\mathbf{P}^{-} \text {invariant }}$ function on $\mathfrak{g}$.

Proof. Let $g, \Gamma$ be as in Proposition 3.1 and let $\mathbf{p}$ belong to $\mathbf{P}$. Factor $\mathbf{p} \Gamma^{-1}$ into the product $\mathbf{p} \Gamma^{-1}=\Gamma_{1}^{-1} \mathbf{g}^{\prime}$, where $\Gamma_{1} \in \exp \left(\tilde{\mathcal{F}}_{+}\right)$and $\mathbf{g}^{\prime} \in \mathbf{G}^{\prime}$. Then $A d_{\mathbf{p}} g=$ $A d_{\Gamma_{1}^{-1} \mathbf{g}^{\prime}}\left(\lambda e_{-m}+P(g)\right)=A d_{\Gamma_{1}^{-1}}\left(\lambda e_{-m}+A d_{\mathbf{g}^{\prime}} P(g)\right)$. Then it follows from Proposition 3.1 that $P\left(A d_{\mathbf{p}} g\right)=A d_{\mathbf{g}^{\prime}} P(g)$ and, therefore, $f\left(P\left(A d_{\mathbf{p}} g\right)\right)=f(P(g))$. 
Consider now algebra $\mathcal{A}^{\prime}$ of $A d_{\mathbf{B}_{+}^{\prime}}$-invariant functions on $\mathfrak{g}^{\prime}$ constructed in the same way as $\mathcal{A}$ on $\mathfrak{g}$. Then, by Theorem $4.1 \mathcal{A}^{\prime} \circ \phi_{1}$ is a Poisson subalgebra of $\mathcal{A}$. The same is true for $\mathcal{A}_{\mathcal{P}_{+}} \circ P$, where $A_{\mathcal{P}_{+}}$is an algebra of $A d_{\mathbf{G}^{\prime}}$-invariant functions on $\mathcal{P}_{+}$. Moreover, it follows from Lemmas 5.1, 5.2 that

$$
\left\{f_{1} \circ P, f_{2} \circ \phi_{1}\right\}=0
$$

for any $f_{1} \in \mathcal{A}_{\mathcal{P}_{+}}, f_{2} \in \mathcal{A}^{\prime}$.

Let $\mathcal{A}_{1}$ be a Poisson subalgebra of $\mathcal{A}$ generated by $\mathcal{A}^{\prime} \circ \phi_{1}, \mathcal{A}_{\mathcal{P}_{+}} \circ P$. Then Theorem 4.1 and (5.5) imply that functions $I_{j}^{\prime} \circ \phi_{1}, j=1, \ldots, r-1$, where $I_{j}^{\prime}$ are the Chevalley invariants of $\mathfrak{g}^{\prime}$ lie in the center of $\mathcal{A}_{1}$ along with the Chevalley invariants $I_{i}, i=1, \ldots, r$ of $\mathfrak{g}$. This allow us to restrict all the considerations below to the case when both $g$ and $\phi_{1}(g)$ are regular elements of $\mathfrak{g}$ and $\mathfrak{g}^{\prime}$. In particular, functions from $\mathcal{A}_{\mathcal{P}_{+}}$we are going to consider will be defined on $\mathcal{P}_{+}^{r}=\left\{p=g^{\prime}+v_{+}+x_{+} e_{m}: g^{\prime} \in \mathfrak{g}^{\prime}\right.$ is regular; $\left.v_{+} \in \mathcal{V}_{+}\right\}$.

Note that a linear function

$$
l_{m}(p)=x_{+}=\frac{\left\langle e_{-m}, p\right\rangle}{\left\langle e_{-m}, e_{m}\right\rangle}
$$

is $A d_{\mathbf{G}^{\prime}}$-invariant due to $(2.3)$.

To compute the functional dimension of $\mathcal{A}_{1}$ we need

Lemma 5.3. There exist $\left(\operatorname{dim} \mathcal{F}_{+}-r\right)$ functionally independent over $\mathcal{V}_{+} A_{\mathbf{G}^{\prime} \text {-invariant }}$ functions on $\mathcal{P}_{+}^{r}$.

Proof. Write $p \in \mathcal{P}_{+}^{r}$ as $p=g^{\prime}+v+x_{+} e_{m}$, where $g^{\prime}=A d_{\mathbf{g}^{\prime}} h^{\prime}$ and $h^{\prime}$ is a regular element of the Cartan subalgebra $\mathfrak{h}^{\prime}$ of $\mathfrak{g}^{\prime}$. Then $\mathbf{g}^{\prime}$ is defined uniquely up to the right multiplication by an element of the maximal torus $\mathbf{T}^{\prime}$ of $\mathfrak{g}^{\prime}$.

Let $\mathbf{k}=\left(k_{\alpha}\right)_{\alpha \in \Phi_{\mathcal{F}}^{+}}$be a nonnegative integral solution of the equation

$$
\sum_{\alpha \in\left(\Phi_{\mathcal{F}}^{+} \backslash\{m\}\right)} k_{\alpha} \alpha=k_{m} m .
$$

Then, since $m\left(\mathfrak{h}^{\prime}\right)=0$, the monomial

$$
\tilde{f}_{\mathbf{k}}(v)=\Pi_{\alpha \in\left(\Phi_{\mathcal{F}}^{+} \backslash\{m\}\right)}\left\langle v, e_{-\alpha}\right\rangle
$$

is an $A d_{\mathbf{T}^{\prime}}$-invariant function on $\mathcal{V}_{+}$and, therefore function

$$
f_{\mathbf{k}}(p)=\tilde{f}_{\mathbf{k}}\left(A d_{\mathbf{g}^{\prime}}^{-1} v\right)
$$

is $A d_{\mathbf{G}^{\prime}}$-invariant. The number of independent monomials $\tilde{f}_{\mathbf{k}}$ is equal to the number of linearly independent solutions of $(5.7)$, which, in turn, is equal to $\left(\operatorname{dim} \mathcal{F}_{+}-r\right)$.

It should be emphasized that, due to the Remark 3 in the end of section 3.1, functions $f_{\mathbf{k}} \circ P, l_{m} \circ P$ are independent of functions from $\mathcal{A}^{\prime}$. Therefore, Theorem 5.1, Lemma 5.3 and property (5.5) lead us to the following 
Proposition 5.1. The functional dimension of the Poisson subalgebra $\mathcal{A}_{1}$ is equal to $\left(\operatorname{dim} O_{\zeta}-2 r+1\right)$. The center of $\mathcal{A}_{1}$ is generated by $2 r-1$ functions $I_{i}, i=1, \ldots, r$ and $I_{j}^{\prime} \circ \phi_{1}, j=1, \ldots, r-1$.

If $\mathcal{C}^{\prime}$ (resp. $\mathcal{C}_{\mathcal{P}_{+}}$) is a maximal involutive subalgebra of $\mathcal{A}^{\prime}$ (resp. $\mathcal{A}_{\mathcal{P}_{+}}$) then subalgebra $\mathcal{C}$ generated by $\mathcal{C}^{\prime}, \mathcal{C}_{\mathcal{P}_{+}}$is the maximal involutive subalgebra of $\mathcal{A}_{1}$ whose functional dimension is equal to $1 / 2 \operatorname{dim} O_{\zeta}$.

Proposition 5.1 suggests an inductive procedure of constructing the maximal Poisson commutative family of integrals for the Toda flows in $\mathfrak{g}$. Indeed, if we know how to construct $\mathcal{C}_{\mathcal{P}_{+}}$then we are left with the problem of constructing the maximal Poisson commutative subalgebra in $\mathcal{A}^{\prime}$, which is the same as our initial problem but for the algebra $\mathfrak{g}^{\prime}$ of smaller rank.

We shall construct $\mathcal{C}_{\mathcal{P}_{+}}$in sections 7,8 . But before we would like to show that results of this section already enable us to prove the complete integrability of the Toda flows in the particular case when $\mathfrak{g}$ is of type $G_{2}$.

\section{Example: Generic Toda Flows in $G_{2}$ Are Completely Integrable.}

Let $\alpha_{1}$ be the short and $\alpha_{2}$ be the long simple positive roots of $G_{2}$. Then $m=2 \alpha_{2}+3 \alpha_{1}$ and $\Phi_{\mathcal{F}}^{+}=\left\{m, \alpha_{2}+i \alpha_{1}(i=0,1,2,3)\right\} \cdot \mathfrak{g}^{\prime}$ is an algebra of type $A_{1}$ generated by $e_{ \pm \alpha_{1}}, h_{1}$ and the maximal family of strongly orthogonal roots consists of $m$ and $\alpha_{1}$.

According to Theorem 3.1 the dimension of a generic coadjoint orbit in $\epsilon+\mathfrak{b}_{-}$is equal to 8 . Therefore, we need 4 independent integrals in involution. We can choose 3 of them to be the Chevalley invariants $I_{1}, I_{2}$ of $G_{2}$ and a superposition $I_{1}^{\prime} \circ \phi_{1}$ of the Chevalley invariant $I_{1}^{\prime}$ of $A_{1}$ with the 1-chop map $\phi_{1}$.

Let us now choose generators of the algebra $A_{\mathcal{P}_{+}}$of $A d_{\mathbf{G}^{\prime}}$-invariant functions on $\mathcal{P}_{+}$. Denote

$$
v_{i}(v)=\left\langle e_{\alpha_{2}+i \alpha_{1}}, v\right\rangle(i=0,1,2,3) .
$$

Then functions

$$
\tilde{f}_{1}(v)=v_{0} v_{3}, \tilde{f}_{2}(v)=v_{1} v_{2}, \tilde{f}_{3}(v)=v_{0} v_{2}^{3}
$$

form a maximal family of independent $\mathfrak{h}^{\prime}$-invariant functions on $\mathcal{V}_{+}$. By (5.6), (5.9), $A_{\mathcal{P}_{+}}$ is generated by $f_{i}(p)=\tilde{f}_{i}\left(A d_{\mathbf{g}^{\prime}} v\right), i=1,2,3$ and functions $l_{m}(p)$ and $I_{1}^{\prime}\left(g^{\prime}\right)$. Here $g^{\prime}, \mathbf{g}^{\prime}$ and $v$ are defined as in the proof of Lemma 5.3.

Due to (5.5), $I_{1}^{\prime} \circ \phi_{1}$ is involution with any function from $A_{\mathcal{P}_{+}} \circ P$. The same is true for $I_{1}, I_{2}$. However, the functional dimension of $A_{\mathcal{P}_{+}} \circ P$ is 5 and, therefore, any function $J$ from $A_{\mathcal{P}_{+}} \circ P$ which is independent with $I_{1}, I_{2}, I_{1}^{\prime} \circ \phi_{1}$ can be used as the fourth integral needed for complete integrability and, moreover, if we pick another function, $\tilde{J}$, as the fourth integral, the Poisson bracket of $J$ and $\tilde{J}$ does not have to be zero. In particular, $J$ can be chosen to be either one of the functions $f_{i} \circ P$ obtained from (6.1).

Finally, we present another choice of the fourth integral $J$ that does not involve the adjoint action of $\mathbf{g}^{\prime}$. Recall (Proposition 2.1) that the adjoint action of $\mathbf{G}^{\prime}$ on $\mathcal{V}_{+}$preserves a nondegenerate skewsymmetric form $\left\langle a d_{e_{m}}, \cdot \cdot\right\rangle$ that endows $\mathcal{V}_{+}$with a constant symplectic structure. The action of $\mathbf{G}^{\prime}$ is Hamiltonian. For an element $g^{\prime} \in \mathfrak{g}^{\prime}$ the flow of $A d_{\exp \left(g^{\prime} t\right.}$ is a Hamiltonian flow generated by $H_{g^{\prime}}(v)=\frac{1}{2}\left\langle a d_{e_{-m}} a d_{g^{\prime}} v, v\right\rangle$. Then the moment map 
$\mathcal{J}: \mathcal{V}_{+} \rightarrow \mathfrak{g}^{\prime}$ is given by

$$
\mathcal{J}(v)=\frac{1}{2} \pi_{\mathfrak{g}^{\prime}}\left(\left[v, a d_{e_{-m}} v\right]\right)=\frac{1}{2}\left(\left[v, a d_{e_{-m}} v\right]\right) .
$$

(The last equality can be derived from (2.3).)

It follows that function

$$
f(p)=f(v)=\left\langle\left[a d_{e_{-m}} v, v\right],\left[a d_{e_{-m}} v, v\right]\right\rangle .
$$

is $A d_{\mathrm{G}^{\prime}}$-invariant. In the case of $G_{2}$, a direct computation shows that function $J=f \circ P$ is nontrivial and idependent with $I_{1}, I_{2}, I_{1}^{\prime} \circ \phi_{1}$. Invoking (5.5), we conclude that functions $I_{1}, I_{2}, I_{1}^{\prime} \circ \phi_{1}, J$ form a maximal family of independent integrals in involution.

\section{InVOLUtive Integrals}

Recall that in Proposition 4.1 we defined a Poisson bracket $\{,\}^{\lambda}$ on $\mathcal{P}_{+}$as a pushforward of the Lie-Poisson bracket on $\mathfrak{g}$ under the map $P$. In this section we construct a family of $A d_{\mathbf{G}^{\prime}}$-invariant and Poisson commutative with respect to $\{,\}^{\lambda}$ functions on $\mathcal{P}_{+}$. Then, by Proposition 4.1 and Lemma 5.2, a composition with the map $P$ will give us an involutive family of $A d_{\mathcal{P}_{+}}$-invariant integrals of the Toda flow.

In our construction we utilize the idea of the shift of argument that was introduced by Manakov [Ma] and then developed by Mischenko and Fomenko [MiFo2]. In these works the "shifts" $I_{\mu, a}(g)=I(g+\mu a)$ in the direction of a generic vector $a$ were used to construct a maximal Poisson commutative family of functions on adjoint orbits in semisimple algebras. Two important distinctions in our case are that, firstly, functions $I_{\mu, a}$ are not constant along the Toda flows and so we have first to apply a nonlinear map $P$ to $g$, and, secondly, we use the shift of argument in a "maximally nongeneric" direction of $e_{m}$.

Namely, for an $A d_{\mathbf{G}}$-invariant function $I \in C^{\infty}(\mathfrak{g})$ we define a function $I_{\mu} \in C^{\infty}\left(\mathcal{P}_{+}\right)$ by

$$
I_{\mu}(p)=I\left(\lambda e_{-m}+p+\mu e_{m}\right) .
$$

Denote

$$
p_{\mu}=\lambda e_{-m}+p+\mu e_{m}=\lambda e_{-m}+g^{\prime}+v+\left(x_{+}+\mu\right) e_{m} .
$$

Then the equality

$$
\left[\nabla I\left(p_{\mu}\right), p_{\mu}\right]=0
$$

and (2.3), (2.6), (2.7) imply

$$
\begin{aligned}
& \left\langle\nabla I\left(p_{\mu}\right), h_{m}\right\rangle=0 \\
& {\left[\pi_{\mathcal{V}_{+}} \nabla I\left(p_{\mu}\right), v\right]=0 .}
\end{aligned}
$$

Note also that

$$
\nabla I_{\mu}(p)=\pi_{\mathcal{P}_{-}} \nabla I\left(p_{\mu}\right) .
$$

Let $\{,\}^{\lambda}$ be the Poisson bracket defined by (4.7), (4.8). 
Theorem 7.1. Let $I, J$ be $A d_{G}$-invariant functions. Then for any $\mu$ and $\nu$

$$
\left\{I_{\mu}, J_{\nu}\right\}^{\lambda}=0 .
$$

Proof. First, consider $\left\{I_{\mu}, J_{\nu}\right\}_{0}$. Since, by (7.2), (7.3)

$$
\begin{aligned}
{\left[p, \pi_{\mathcal{P}_{-}} \nabla I\left(p_{\mu}\right)\right] } & =\left[p, \nabla I\left(p_{\mu}\right)\right]-\left[p, \pi_{\mathcal{V}_{+}} \nabla I\left(p_{\mu}\right)\right] \\
& =-\mu\left[e_{m}, \nabla I\left(p_{\mu}\right)\right]-\lambda\left[e_{-m}, \nabla I\left(p_{\mu}\right)\right]-\left[g^{\prime}, \pi_{\mathcal{V}_{+}} \nabla I\left(p_{\mu}\right)\right],
\end{aligned}
$$

we have

$$
\begin{aligned}
\left\{I_{\mu}, J_{\nu}\right\}_{0}(p) & =\left\langle\left[p, \pi_{\mathcal{P}_{-}} \nabla I\left(p_{\mu}\right)\right], \pi_{\mathcal{P}_{-}} \nabla J_{\nu}\left(p_{\nu}\right)\right\rangle \\
& =-\mu\left\langle\left[e_{m}, \nabla I\left(p_{\mu}\right)\right], \pi_{\mathcal{P}_{-}} \nabla J_{\nu}\left(p_{\nu}\right)\right\rangle+\left\langle\left[\pi_{\mathcal{V}_{+}} \nabla I\left(p_{\mu}\right), g^{\prime}\right], \pi_{\mathcal{P}_{-}} \nabla J_{\nu}\left(p_{\nu}\right)\right\rangle \\
& =-\mu\left\langle e_{m},\left[\nabla I\left(p_{\mu}\right), \nabla J_{\nu}\left(p_{\nu}\right)\right]\right\rangle+\left\langle\pi_{\mathcal{V}_{+}} \nabla I\left(p_{\mu}\right),\left[g^{\prime}, \pi_{\mathcal{P}_{-}} \nabla J_{\nu}\left(p_{\nu}\right)\right]\right\rangle \\
& =-\mu\left\langle e_{m},\left[\nabla I\left(p_{\mu}\right), \nabla J_{\nu}\left(p_{\nu}\right)\right]\right\rangle+\left\langle\pi_{\mathcal{V}_{+}} \nabla I\left(p_{\mu}\right),\left[g^{\prime}, \nabla J_{\nu}\left(p_{\nu}\right)\right]\right\rangle .
\end{aligned}
$$

But, due to (7.2)

$$
\left[g^{\prime}, \nabla J_{\nu}\left(p_{\nu}\right)\right]=-\left[\nu e_{m}+v, \nabla J_{\nu}\left(p_{\nu}\right)\right]-\lambda\left[e_{-m}, \nabla J_{\nu}\left(p_{\nu}\right)\right]
$$

and by (2.3), (7.3)

$$
\left[\pi_{\mathcal{V}_{+}} \nabla I\left(p_{\mu}\right), \nu e_{m}+v\right]=0 .
$$

Therefore,

$$
\begin{aligned}
\left\{I_{\mu}, J_{\nu}\right\}_{0}(p) & =-\mu\left\langle e_{m},\left[\nabla I\left(p_{\mu}\right), \nabla J_{\nu}\left(p_{\nu}\right)\right]\right\rangle-\lambda\left\langle\pi_{\mathcal{V}_{+}} \nabla I\left(p_{\mu}\right),\left[e_{-m}, \nabla J_{\nu}\left(p_{\nu}\right)\right]\right\rangle \\
& =\left\langle-\mu e_{m}+\lambda e_{-m},\left[\nabla I\left(p_{\mu}\right), \nabla J_{\nu}\left(p_{\nu}\right)\right]\right\rangle .
\end{aligned}
$$

On the other hand, similar considerations show that

$$
\left\{I_{\mu}, J_{\nu}\right\}_{0}(p)=\left\langle-\nu e_{m}+\lambda e_{-m},\left[\nabla I\left(p_{\mu}\right), \nabla J_{\nu}\left(p_{\nu}\right)\right]\right\rangle .
$$

Comparing the last two equations we obtain

$$
\left\langle e_{m},\left[\nabla I\left(p_{\mu}\right), \nabla J_{\nu}\left(p_{\nu}\right)\right]\right\rangle=0
$$

and

$$
\left\{I_{\mu}, J_{\nu}\right\}_{0}(p)=\lambda\left\langle e_{-m},\left[\nabla I\left(p_{\mu}\right), \nabla J_{\nu}\left(p_{\nu}\right)\right]\right\rangle .
$$

Now consider $\left\{I_{\mu}, J_{\nu}\right\}_{1}$. Due to $(7.4),(7.6)$

$$
\begin{aligned}
& \pi_{\mathcal{V}_{-}}\left[\nabla I_{\mu}(p), p\right]=\lambda\left[e_{-m}, \nabla I\left(p_{\mu}\right)\right] \\
& \pi_{\mathcal{V}_{-}}\left[\nabla J_{\nu}(p), p\right]=\lambda\left[e_{-m}, \nabla J\left(p_{\nu}\right)\right] .
\end{aligned}
$$

Therefore,

$$
\begin{aligned}
\left\{I_{\mu}, J_{\nu}\right\}_{1}(p) & =\lambda^{2}\left\langle\left[e_{m},\left[e_{-m}, \nabla I\left(p_{\mu}\right)\right]\right],\left[e_{-m}, \nabla J\left(p_{\nu}\right)\right]\right\rangle \\
& =\lambda^{2}\left\langle\nabla I\left(p_{\mu}\right),\left[e_{-m}, \nabla J\left(p_{\nu}\right)\right]\right\rangle \\
& =-\lambda^{2}\left\langle e_{-m},\left[\nabla I\left(p_{\mu}\right), \nabla J\left(p_{\nu}\right)\right]\right\rangle .
\end{aligned}
$$

Finally, it follows form (7.7), (7.8) that

$$
\left\{I_{\mu}, J_{\nu}\right\}^{\lambda}(p)=\left\{I_{\mu}, J_{\nu}\right\}_{0}(p)+\lambda^{-1}\left\{I_{\mu}, J_{\nu}\right\}_{1}(p)=0 .
$$

To conclude this section, we prove the following 
Lemma 7.1. Let $l_{m}(p)$ be the function defined by (5.6). Then

(i) $\left\{l_{m}, f\right\}_{0}=0$ for any $f \in C^{\infty}\left(\mathcal{P}_{+}\right)$.

(ii) $\left\{l_{m}, f\right\}_{1}=0$ for any $\mathbf{G}^{\prime}$-invariant $f \in C^{\infty}\left(\mathcal{P}_{+}\right)$.

Proof. (i) follows from the equality $\left[e_{-m}, \nabla f\right]=0$

To prove (ii), recall that if $f$ is $\mathbf{G}^{\prime}$-invariant then $\left\langle\left[\nabla f, g^{\prime}\right], p\right\rangle=0$ for any $g^{\prime} \in \mathfrak{g}^{\prime}$. Let $p=g^{\prime}+v+x_{+} e_{m}$. Then

$$
\begin{aligned}
& \left\langle a d_{e_{m}} \pi_{\mathcal{V}_{-}}[\nabla f(p), p], \pi_{\mathcal{V}_{-}}\left[e_{-m}, p\right]\right\rangle=-\left\langle\pi_{\mathcal{V}_{-}}[\nabla f(p), p],\left[e_{m},\left[e_{-m}, p\right]\right]\right\rangle \\
& =\langle[p, \nabla f(p)], v\rangle=\left\langle[p, \nabla f(p)], g^{\prime}+v\right\rangle \\
& =\left\langle\nabla f(p),\left[g^{\prime}+v, p\right]\right\rangle=\langle\nabla f(p),[p, p]\rangle=0 .
\end{aligned}
$$

\section{INDEPENDENCE}

Let $I_{1}, \ldots, I_{r}$ be polynomials that generates the algebra of $A d_{G}$-invariant functions. Consider polynomial functions $I_{1 \mu}, \ldots, I_{r \mu}$ defined by (7.1). Then

$$
I_{i \mu}(p)=I_{i}\left(p_{\mu}\right)=\sum_{j=0}^{n_{i}} I_{i j}(p) \mu^{j}
$$

By Theorem 7.1, functions $I_{i j}$ are in involution w.r.t. $\{,\}^{\lambda}$.

Let $p=g^{\prime}+v+x_{+} e_{m}$. We want to prove

Theorem 8.1. For generic fixed $g^{\prime}$ and $x_{+}$we can find among $I_{i j}$ considered as functions on $\mathcal{V}_{+}$at least $N=\frac{1}{2} \operatorname{dim} \mathcal{V}_{+}$almost everywhere independent $f$ unctions.

Note first that it is sufficient to prove this theorem for $x_{+}=0$ and $g^{\prime}=h$, where $h$ belongs to the Cartan subalgebra $\mathfrak{h}^{\prime}$ of $\mathfrak{g}^{\prime}$. Moreover, it suffices to show that there exist such $v \in \mathcal{V}_{+}$that

$$
\operatorname{dim} \operatorname{Span}\left\{\pi_{\mathcal{V}_{-}} \nabla I_{i j}(p)\right\} \geq N .
$$

¿From now on we fix $\lambda$ to be 1 . Recall, that $I_{1}(g)=\langle g, g\rangle$ and, in particular, $1 / 2 \nabla I_{1}\left(p_{\mu}\right)=p_{\mu}=e_{m}+p+\mu e_{m}$. We can choose $c_{i}, i=2, \ldots, r$ in such a way that

$$
\left\langle\nabla I_{i}\left(p_{\mu}\right)-c_{i} p_{\mu}, e_{m}\right\rangle=0
$$

Then

$$
Y_{i}\left(p_{\mu}\right)=\nabla I_{i}\left(p_{\mu}\right)-c_{i} p_{\mu}, i=2, \ldots, r
$$

satisfy

$$
\left[Y_{i}\left(p_{\mu}\right), p_{\mu}\right]=0
$$

Due to $(8.1)$,

$$
Y_{i}\left(p_{\mu}\right)=\sum_{j=0}^{n_{i}} X_{i j}(p) \mu^{j}
$$


where $X_{i j}(p)$ are polynomial $\mathfrak{g}$-valued functions of $v$. Then

$$
\pi_{\mathcal{V}_{-}} \nabla I_{i j}(p)=\pi_{\mathcal{V}_{-}} X_{i j}(p)
$$

and (7.2) is equivalent to the system of equations (cf. [Fo], sec 7.5.1)

$$
\begin{aligned}
& {\left[X_{i 0}(p), e_{-m}+p\right]=0} \\
& {\left[X_{i j}(p), e_{-m}+p\right]=\left[e_{m}, X_{i, j-1}(p)\right] \quad j=1, \ldots, n_{i}} \\
& {\left[e_{m}, X_{i, n_{i}}(p)\right]=0}
\end{aligned}
$$

By (7.3), (8.3) we have

$$
\begin{aligned}
& \left\langle X_{i j}, h_{m}\right\rangle=0 \\
& \left\langle X_{i j}, e_{m}\right\rangle=0 \\
& {\left[\pi_{\mathcal{V}_{+}} X_{i j}, v\right]=0 .}
\end{aligned}
$$

Also, it follows from (8.4) that

$$
\begin{aligned}
& {\left[\pi_{\mathcal{V}_{+}} X_{i j}(p), e_{-m}\right]+\left[\pi_{\mathcal{V}_{-}} X_{i j}(p), h\right]=0} \\
& {\left[\pi_{\mathcal{V}_{+}} X_{i j}(p), h\right]+\left[\pi_{\mathfrak{g}^{\prime}} X_{i j}(p), v\right]=\left[e_{m}, \pi_{\mathcal{V}_{-}} X_{i, j-1}(p)\right]}
\end{aligned}
$$

Note that first of the equations (8.5) can be rewritten as

$$
\pi_{\mathcal{V}_{+}} X_{i j}(p)=-a d_{e_{m}} a d_{h} \pi_{\mathcal{V}_{-}} X_{i j}(p)
$$

Let us now represent each $X_{i j}(p)$ as a sum

$$
X_{i j}(p)=\sum_{k} X_{i j}^{k}(p)
$$

where $X_{i j}^{k}(p)$ is a homogeneous $\mathfrak{g}$-valued polynomial of degree $k$.

Lemma 8.1. $X_{i j}^{0}(p)=\pi_{\mathfrak{g}^{\prime}} X_{i j}^{0}(p)$. Moreover, $X_{i 0}^{0}(p), i=2, \ldots, r$ generate $\mathfrak{h}^{\prime}$.

Proof. It is not hard to see that for generic $h$ element $p_{0 \mu}=e_{-m}+h+\mu e_{m}$ is a regular element of $\mathfrak{g}$. Therefore, $\nabla I_{i}\left(p_{0 \mu}\right), i=1, \ldots, r$ span a stabilizer of $p_{0 \mu}$ in $\mathfrak{g}$ which coincides with $\mathfrak{h}^{\prime}+\mathbb{C}\left\{e_{-m}+\mu e_{m}\right\}$. Then $Y_{i}\left(p_{0 \mu}\right)$ span $\mathfrak{h}^{\prime}$. Moreover, since the algebra of $A d_{G^{-}}$ invariant functions can be generated by trace polynomial of the adjoint representation and since $\operatorname{Tr}\left(a d_{e_{-m}+h}^{n}\right)=\operatorname{Tr}\left(a d_{e_{-m}}^{n}\right), X_{i 0}^{0}(p), i=2, \ldots, r$ also span $\mathfrak{h}^{\prime}$.

Let us now consider

$$
L_{i j}:=\pi_{\mathcal{V}_{-}} X_{i j}^{1}(p) .
$$

Due to Lemma 8.1, (8.5) and (8.6),

$$
\begin{aligned}
& a d_{e_{m}} a d_{h}^{2} L_{i j}=a d_{e_{m}} L_{i, j-1}-\left[X_{i j}^{0}, v\right], j=1, \ldots, n_{i} \\
& a d_{e_{m}} a d_{h}^{2} L_{i 0}=-\left[X_{i 0}^{0}(p), v\right] .
\end{aligned}
$$


or

$$
\begin{aligned}
L_{i 0} & =-a d_{h}^{-2}\left[X_{i 0}^{0}, v_{-}\right] \\
L_{i j} & =a d_{h}^{-2}\left(L_{i, j-1}-\left[X_{i j}^{0}, v_{-}\right]\right), j=1, \ldots, n_{i},
\end{aligned}
$$

where $v_{-}=a d_{e_{-m}} v$ ( here we used the fact that $X_{i j}^{0}(p) \in \mathfrak{h}^{\natural}$ and $a d_{e_{-m}}$ commutes with $\left.a d_{\mathfrak{g}^{\prime}} \cdot\right)$

We denote $\left[X_{i j}^{0}, v_{-}\right]$by $w_{i j}$ and the restriction of $a d_{h}^{-2}$ on $\mathcal{V}_{-}$by $A$. Then (8.8) can be rewritten as

$$
L_{i j}=-\sum_{l=0}^{j} A^{l+1} w_{i, j-l} .
$$

It follows form Lemma 8.1 that $L_{i 0}, i=2, \ldots, r$ are independent and each $w_{i j}, j \geq 1$ is a linear combination of $w_{i 0}, i=2, \ldots, r$. If $V_{-}=a d_{\mathfrak{h}^{\prime}} v_{-}$then (8.9) implies

$$
\operatorname{Span}\left\{L_{i j}\right\}=\operatorname{Span}\left\{A^{l} V_{-}, l \geq 1\right\} .
$$

Now we can finish

Proof of Theorem 8.1. Recall (Proposition 2.1) that each weight space of $\mathfrak{h}^{\prime}$ in $\mathcal{V}_{-}$is spanned by the vector $e_{\alpha}, \alpha \in \Phi_{\mathcal{F}}^{-}, \alpha \neq-m$. The highest weight vector is $e_{-\alpha_{i_{0}}}$ and the lowest weight vector is $e_{\alpha_{i_{0}}-m}$, where $\alpha_{i_{0}}$ is the only simple root connected with $-m$ in the extended Dynkin diagram of $\mathfrak{g}$. (As before, we assume that $\mathfrak{g}$ is not of type $A_{r}$.)

Since $(m-\alpha)(h)=-\alpha(h)$ for any $h \in \mathfrak{h}^{\prime}, a d_{\mathfrak{h}^{\prime}}$ acts by diagonal matrices skew-symmetric w.r.t. the antidiagonal. Therefore, for generic $h, A$ acts by the diagonal matrix symmetric w.r.t. the antidiagonal and first $N$ diagonal entries of $A$ are nonzero and distinct.

We may assume that all coordinates of $v_{-}$w.r.t the weight decomposition are nonzero. Then one can find vector $v_{-}^{\prime} \in V_{-}=a d_{\mathfrak{h}^{\prime}} v_{-}$with all coordinates nonzero. Then the standard considerations involving Vandermonde determinants show that $\operatorname{Span}\left\{A^{l} v_{-}^{\prime}, l \geq\right.$ $1\}$ is $N$-dimensional, which, in view of (8.7), (8.10) proves the theorem.

\section{Proof of Main Theorem}

We proceed by induction. Assume that for simple algebras of rank less then $r$ we constructed the maximal Poisson commuting family of integrals of the Toda flow on generic coadjoint orbits.

Let $O_{\zeta_{0}}$ be a generic coadjoint orbit in $\epsilon+\mathfrak{b}_{-} \subset \mathfrak{g}$, where $\zeta_{0}$ is a normal form of the orbit given by (3.3) and let $f_{1}, \ldots, f_{n}$ be functions on $\mathfrak{g}^{\prime}$ whose restrictions to generic orbits in $\epsilon^{\prime}+\mathfrak{b}_{-}^{\prime}$ provide a maximal family of integrals for the Toda flows. For $\zeta=$ $\epsilon+x_{-} e_{-m}+x_{0} h_{m}+v_{-}+\zeta^{\prime} \in O_{\zeta_{0}}$, where $v_{-} \in \mathcal{V}_{-}$and $\zeta^{\prime} \in \mathfrak{b}_{-}^{\prime}$, consider $\phi_{1}(\zeta)$. If $v_{-}=0$ then, by (3.2) and the definition of the 1-chop $\phi_{1}, \phi_{1}(\zeta)$ is equal to $\pi_{\mathfrak{g}^{\prime}} \zeta$ and belongs to a generic orbit in $\epsilon^{\prime}+\mathfrak{b}_{-}^{\prime}$. This ensures that functions $f_{1} \circ \phi_{1}, \ldots, f_{n} \circ \phi_{1}$ are almost everywhere independent on $O_{\zeta_{0}}$.

Due to Proposition 2.2 and Theorem 4.1

$$
\left\{f_{i} \circ \phi_{1}, f_{j} \circ \phi_{1}\right\}_{\epsilon+\mathfrak{b}_{-}}=0,
$$


while Theorem 7.1, Proposition 4.1 and (5.5) imply that

$$
\left\{I_{i j} \circ P, f_{k} \circ \phi_{1}\right\}_{\epsilon+\mathfrak{b}_{-}}=0
$$

where functions $I_{i j}$ are given by (8.1). Futhermore, by Lemma 7.1, the function $l_{m} \circ P$, where $l_{m}$ is defined by (5.6) is in involution with all $I_{i j} \circ P$ and $f_{k} \circ \phi_{1}$.

Let $I_{i_{q} j_{q}}, q=1, \ldots, N$ be independent over $\mathcal{V}_{+}$functions among $I_{i j}$ whose existence is guaranteed by Theorem 8.1. Remark 3 of section 3.1 shows that functions $l_{m} \circ P ; I_{i_{q} j_{q}} \circ$ $P, q=1, \ldots, N ; f_{k} \circ \phi_{1}, k=1, \ldots, n$ are independent on $\epsilon+\mathfrak{b}_{-}$. Ivoking Proposition 3.3, we see that the number of functions in this family is equal to $\frac{1}{2} \operatorname{dim} O_{\zeta_{0}}$ which concludes the proof of Theorem 1.1.

\section{REFERENCES}

[A] Arhangelskij, A. A.: Completely integrable Hamiltonian systems on the group of triangular matrices. Mat. Sb. 108, 134-142 (1979)

[BG] Bloch, A. M. and Gekhtman, M. I. : Hamiltonian and gradient structures in the Toda flows. In preparation.

[DLNT] P. Deift, L. Li, C. Nanda, C. Tomei: The Toda flow on a generic orbit is integrable. Comm. Pure \& Appl. Math., 39, 183-232 (1986)

[EFS] Ercolani, N. M. Flaschka, H. , Singer, S. F.: The geometry of the full Kostant-Toda lattice. Progr. Math., 115, 181-225 (1993)

[F] Flaschka, H.: The Toda lattice I. Phys. Rev. B 9, 1924-1925 (1974)

[Fo] Fomenko, A. T.: Symplectic geometry. Advanced Studies in Contemporary Mathematics, 5. New York: Gordon and Breach Science Publishers 1988

[H] Humphreys, J. E.: Introduction to Lie Algebras and Representation Theory. Berlin Heidelberg New York: Springer 1980

[J] Joseph, A.: A preparation theorem for the prime spectrum of a semisimple Lie algebra. J. Algebra 48, 241-289 (1977)

[K1] Kostant, B.: The solution to a generalized Toda lattice and representation theory. Adv. Math. 34, 195-338 (1979)

[K2] Kostant, B.: The principal three-dimensional subgroup and the Betti numbers of a complex simple Lie groups. Amer. J. Math. 81, 973-1032 (1959)

[K3] Kostant, B.: Lie group representations on polynomial rings. Amer. J. Math. 85, 327-404 (1963)

[LW] Lipsman, R. L. and Wolf, J. A.: Canonical semi-invariants and the Plancherel formula for parabolic groups. Trans. AMS 269, 111-131 (1982)

[Ma] Manakov, S. V.: Note on the integration of Euler's equations of the dynamics of an $n$-dimensional rigid body. Funct. Anal. Appl. 10, 328-329 (1977)

[MiFo1] Mischenko, A. S. and Fomenko, A. T.: A generalized Liouville method for the integration of Hamiltonian systems. Functional Analysis and its Applications 12, 113-131 (1978)

[MiFo2] Mischenko, A. S. and Fomenko, A. T.: Euler equation on finite-dimensional Lie groups. Izvestija 12, 371-389 (1978)

[Mo] Moser, J.: Three integrable Hamiltonian systems connected with isospectral deformation. Adv. Math. 16, 197-220 (1975)

[N] Nehoroshev, N. N. : Action-angle variables and their generalizations. Trans. Moscow Math. Soc. 26, 180-197 (1972)

[OP] Ol'shanetsky, M.A. and Perelomov, A.M. : Explicit solutions of classical generalized Toda models. Invent. Math. 54, 261-269 (1979)

[OV] Onishchik, A. L. and Vinberg, E. B. : Lie Groups and Algebraic Groups. Berlin Heidelberg New York: Springer 1990

[RSTS1] Reyman A.G., Semenov-Tian-Shansky, M. A.: Reduction of Hamiltonian systems, affine Lie algebras and Lax equations I. Invent. Math. 54, 81-100 (1979) 
[RSTS2] Reyman A.G., Semenov-Tian-Shansky, M. A.: Group-theoretical methods in the theory of finitedimensional integrable systems, Dynamical Systems VII, Encyclopaedia of Mathematical Sciences 16, Berlin Heidelberg New York: Springer 1994

[S1] Singer, S.: Ph. D. Dissertation. Courant Institute, 1991.

[S2] Singer, S.: Some maps from the full Toda lattice are Poisson. Phys. Lett. A 174, 66-70 (1993)

[Sy] Symes, W.W.: Systems of Toda type, inverse spectral problems, and representation theory. Invent. Math. 59, 13-51 (1980)

[T] Thimm, A.: Integrable Hamiltonian systems on homogeneous spaces. Ergod. Th. and Dynam. Systems 1, 495-517 (1981)

[Tr1] Trofimov, V. V.: Euler equations on Borel subalgebras of semisimple Lie algebras. Izvestija 43, 714-732 (1979)

[Tr2] Trofimov, V. V.: Finite-dimensional representations of Lie algebras and completely integrable systems. Mat. Sb. 11, 610-621 (1980) 